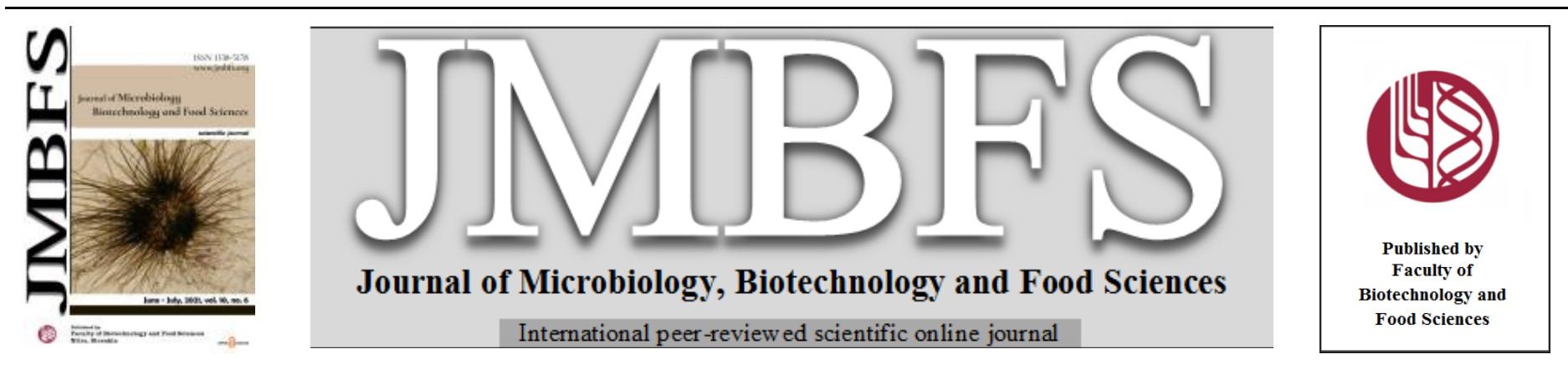

\title{
THE IMPACT OF DIFFERENT PACKAGING AND STORAGE TIME ON PHISYCOCHEMICAL PROPERTIES AND COLOR OF RED WINES
}

\author{
Nebojša Kojić*1, Lidija Jakobek ${ }^{2}$ \\ Address(es): BSc. Nebojša Kojić, \\ ${ }^{1}$ Vupik plus d.o.o., Food Industry, Sajmište 113c, HR-32000 Vukovar, Croatia, +385 998273182 \\ ${ }^{2}$ Josip Juraj Strossmayer University of Osijek, Faculty of Food Technology Osijek, Department of Applied Chemistry and Instrumental Methods, F. Kuhača 20, HR- \\ 31000 Osijek, Croatia.
}

*Corresponding author: nkojic@ptfos.hr

https://doi.org/10.15414/jmbfs.3036

ARTICLE INFO

Received 24. 4. 2020

Revised 9. 12. 2020

Accepted 17. 12. 2020

Published 1. 6. 2021

Regular article

OPEN $\partial_{\text {ACCESS }}$

\section{ABSTRACT}

Packaging and storage time are very important factors that can significantly affect the physicochemical properties of wine and wine quality. The aim of this study was to investigate physicochemical properties and color of wine packed in three different packaging materials (glass, PET (polyethylene terephthalate) and multilayer containers), during the storage period of one year (3, 6, 9 and 12 months). The results showed that during the period of one year, total $\mathrm{SO}_{2}$, free $\mathrm{SO}_{2}$ and oxygen amount decreased, while other properties of wine were constant. Color changed during one year of storage too (yellow color, color intensity and hue increased, while red and blue percentage decreased). In glass and PET containers those changes were less pronounced.

Keywords: packaging, phisyco-chemical properties, oxygen, color

\section{INTRODUCTION}

Packaging of wine is an important factor for wine quality evolution. Usually wine is packed in glass containers but in recent years PET containers (polyethylene terephthalate) have become an option for packaging. They can be produced as single-layer or multi-layer containers (Ghidossi et al., 2012). Other packagings of wine include bag-in-box or multilayer packaging. Bag-in-box packaging consists of a bag (single or multilayer) and an outer box or a container (Robertson, 1993). A multilayer packaging consists of several layers, usually plastic, aluminum foil and paper. One of the main characteristics of packaging that affects the quality of wine is the transfer of gases through the packaging material (Ghidossi et al., 2012). Gases can permeate through the packaging material or the closure and affect the wine characteristics (Lopes et al., 2009; Dimkou et al., 2011; Hopfer et al., 2012; Toussaint et al., 2014).

Storage time also affects wine evolution and quality. For example, the storage time affects the evolution of phenolic compounds (Monagas et al., 2005), oxygen amount dissolved in wine, $\mathrm{SO}_{2}$ amount (Dimkou et al., 2011; Ghidossi et al., 2012; Gambuti et al., 2013; Toussaint et al., 2014), sensory characteristics and color (Skouroumounis et al., 2005; Caillé et al., 2010). One of the main parameters that connects the influence of packaging type and storage time on the wine evolution, is the oxygen content in wine.

Namely, oxygen can be present in wine as dissolved oxygen or in the headspace of the packaging container but it can also permeate into the package through the packaging material or the closure. Once inside the package (in the wine), oxygen can cause an oxidation reaction that affects $\mathrm{SO}_{2}$ decrease in wine (Dimkou et al., 2011; Ghidossi et al., 2012), phenolic compound evolution (Gambuti et al., 2013), and color change or other sensory property changes (Skouroumounis et al., 2005; Lopes et al. 2009; Caillé et al., 2010; Dimkou et al., 2011; Ghidossi et al., 2012). All of these changes are time dependent. Therefore, packaging and storage time are important factors controlling the $\mathrm{O}_{2}$ amount after bottling, and consequently the wine quality evolution. Furthermore, the influence of oxygen on the quality of wine can depend on the storage temperature. Wine is usually stored in wine cellars at 10 to $14{ }^{\circ} \mathrm{C}$. But in stores, the storage temperature can be higher. The conditions of temperature can also influence the amount of oxygen in the wine and by that the changes in wine during storage. The influence of oxygen on the wine quality and the influence of packaging and storage time are still not completely understood.

The aim of this work was to study the influence of packaging (glass bottles, PET bottles and multilayer containers), and storage $(0,3,6,9$ and 12 months, room temperature $25^{\circ} \mathrm{C}$ ) on wine characteristics (wine specific weight, distillate specific weight, alcohol percentage, total dry extract, total acids, volatile acids, free and total $\mathrm{SO}_{2}$ ) and color. The study was conducted on four red wines (Cabernet Sauvignon, Frankovka, Merlot, and Pinot noir). Oxygen was measured during the whole storage period.

\section{MATERIAL AND METHODS}

Samples

Grapes from Vitis vinifera L. (Cabernet sauvignon, Frankovka, Merlot and Pinot noir) were cultivated in a vineyard in Baranja County, Croatia. After producing the wine from a 2018 vintage in a winery, wines were packed in glass bottles (Versus, PP neck - Vetropack, Croatia), polyethylene terephthalate bottles (PCO 28, transparent - Keples, Croatia) and multilayer packaging (multilayer paperboard with metallocene PE resin and aluminium foil (> $99 \%$ aluminium) SIG Combibloc, Switzerland). The volume was $0.5 \mathrm{~L}$ for all packages. Wine was stored at a controlled room temperature $25^{\circ} \mathrm{C}$, and analyzed at the time of packaging and after 3, 6, 9 and 12 months of storage. At each time period, new packages were opened for the analysis.

\section{Physico-chemical properties}

All analyses of the physico-chemical properties of wine were performed according to standard procedures of the International Organization of Vine and Wine (OIV, 2019). Alcohol in wine was determined by using OIV-MA-AS31201B. Total acidity (as tartaric acid) was determined by the neutralization method with $0.1 \mathrm{M} \mathrm{NaOH}$ and a bromothymol blue as an indicator. Volatile acids were determined by using OIV-MA-AS313-02. Specific weights were determined by using OIV-MA-AS2-01A. Free and total sulfur dioxide in wine were determined by using the iodometric method by Ripper. Total dry extract was determined by using OIV-MA-AS2-03B. These methods were already described earlier (Kojić and Jakobek, 2019).

\section{Dissolved oxygen measurement}

Determination of dissolved oxygen was carried out using an oximeter (AL20OXY, Aqualytic, Germany). The wine was poured into a laboratory glass, placed on a magnetic stirrer and mixed. The oximeter electrode was calibrated according to the oxygen content of the air. Electrode was then, immersed into the wine and the dissolved oxygen amount $(\mathrm{mg} / \mathrm{L})$ was measured. 


\section{Color measurement}

Measurements were carried out on a UV-VIS spectrophotometer (Perkin Elmer, Lambda 2, Germany) in a $1 \mathrm{~cm}$ thick quartz cuvette versus distilled water as a blank. Using absorbance values at 420, 520 and $620 \mathrm{~nm}$, five chromatic parameters were calculated according to the Glories method (Glories, 1984) These parameters were color intensity $(\mathrm{CI})$, hue $(\mathrm{H})$, percentage of yellow (Yellow \%), percentage of red (Red \%), percentage of blue (Blue \%). These five chromatic parameters were calculated according to

$\mathrm{CI}=(\mathrm{Abs} 420+\mathrm{Abs} 520+\mathrm{A} 620)$

$\mathrm{H}=\mathrm{Abs} 420 / \mathrm{Abs} 520$

Yellow $\%=($ Abs420/CI $) \times 100 \%$

Red $\%=(\mathrm{Abs} 520 / \mathrm{CI}) \times 100 \%$

Blue $\%=($ Abs620/CI $) \times 100 \%$

\section{Statistical analyses}

All results for the physico-chemical parameters and color were analyzed with multiple regression with main effects and two variable interactions using Minitab software (Minitab LLC., State College, PA, USA). The three variables from which regression terms were constructed are type of packaging, time of storage, and type of wine. Contrasts in the variables were defined for the regression to facilitate comparisons, and were typically arranged to be orthogonal contrasts to facilitate the identification of the subset of strongly significant terms $(\mathrm{p}<0.001)$ Some three variable interactions were introduced when called for by residual analysis, so that no residuals had standardized values larger than 3 . The purpose was to find statistically significant differences between wine characteristics and to create a model with fitted values and standard errors (SE) for each wine characteristic measurement.

\section{RESULTS AND DISCUSSION}

The changes in the chemical composition of wine depend on the storage time but also on the packaging material. Because of such changes in the first several months, wines are often packed and stored for a time period of approximately one year (Hopfer et al., 2012). Various wine characteristics may quantify these changes across time, including numerous physicochemical parameters like alcohol percentage, specific weight of wine and distillate, amounts of acids or $\mathrm{SO}_{2}$, and color of wine. In this study four different wines were packed in three different packaging materials during one year and physicochemical parameters were analysed every three months to see the changes in those changes during time and the possible influence of packaging material on those changes. The results for the physicochemical properties of analyzed wines (Cabernet sauvignon, Frankovka, Merlot, Pinot noir) during storage time of 3, 6, 9 and 12 months were shown in table 1 and figures 1 to 4 . The values for specific wine weight $(0.99310-0.99500)$, specific distillate weight $(0.98000-0.98375)$, alcohol percentage $(12.59-15.65)$, total dry extract $(28.7-36.7 \mathrm{~g} / \mathrm{L})$, total acids $(4.94-6.75 \mathrm{~g} / \mathrm{L})$, volatile acids $(0.43-0.76 \mathrm{~g} / \mathrm{L})$, free $\mathrm{SO}_{2}(7.9-41 \mathrm{mg} / \mathrm{L})$ and total $\mathrm{SO}_{2}(13.0-102 \mathrm{mg} / \mathrm{L})$ were in accordance with data from literature (Dimkou et al., 2011; Ghidossi et al., 2012; Hopfer et al., 2012) and with our earlier study (Kojić and Jakobek, 2019).

Table 1 Physico-chemical properties of wine during 12 months of storage at $25^{\circ} \mathrm{C}$

\begin{tabular}{|c|c|c|c|c|c|c|c|c|c|c|c|c|}
\hline \multirow{2}{*}{$\begin{array}{l}\text { Time } \\
\text { (months) }\end{array}$} & \multicolumn{3}{|c|}{ Cabernet sauvignon } & \multicolumn{3}{|c|}{ Frankovka } & \multicolumn{3}{|c|}{ Merlot } & \multicolumn{3}{|c|}{ Pinot noir } \\
\hline & Glass & PET & Multilayer & Glass & PET & Multilayer & Glass & PET & Multilayer & Glass & PET & Multilayer \\
\hline \multicolumn{13}{|c|}{ Specific weight of wine } \\
\hline $\mathbf{0}$ & 0.99410 & 0.99410 & 0.99410 & 0.99435 & 0.99435 & 0.99435 & 0.99460 & 0.99460 & 0.99460 & 0.99375 & 0.99375 & 0.99375 \\
\hline 3 & 0.99415 & 0.99410 & 0.99430 & 0.99415 & 0.99410 & 0.99430 & 0.99480 & 0.99440 & 0.99445 & 0.99355 & 0.99350 & 0.99375 \\
\hline 6 & 0.99430 & 0.99425 & 0.99470 & 0.99425 & 0.99405 & 0.99500 & 0.99470 & 0.99395 & 0.99310 & 0.99390 & 0.99360 & 0.99430 \\
\hline 9 & 0.99425 & 0.99420 & 0.99485 & 0.99415 & 0.99420 & 0.99445 & 0.99430 & 0.99370 & 0.99345 & 0.99350 & 0.99340 & 0.99405 \\
\hline 12 & 0.99410 & 0.99405 & 0.99490 & 0.99455 & 0.99405 & 0.99450 & 0.99470 & 0.99355 & 0.99355 & 0.99385 & 0.99350 & 0.99435 \\
\hline \multicolumn{13}{|c|}{ Specific weight of distillate } \\
\hline 0 & 0.98060 & 0.98060 & 0.98060 & 0.98300 & 0.98300 & 0.98300 & 0.98140 & 0.98140 & 0.98140 & 0.98165 & 0.98165 & 0.98165 \\
\hline 3 & 0.98055 & 0.98050 & 0.98040 & 0.98295 & 0.98275 & 0.98295 & 0.98120 & 0.98115 & 0.98125 & 0.98155 & 0.98150 & 0.98150 \\
\hline 6 & 0.98065 & 0.98020 & 0.98095 & 0.98280 & 0.98260 & 0.98375 & 0.98095 & 0.98065 & 0.98140 & 0.98185 & 0.98125 & 0.98205 \\
\hline 9 & 0.98060 & 0.98000 & 0.98110 & 0.98280 & 0.98240 & 0.98295 & 0.98060 & 0.98045 & 0.98170 & 0.98145 & 0.98140 & 0.98190 \\
\hline 12 & 0.98085 & 0.98000 & 0.98105 & 0.98345 & 0.98240 & 0.98300 & 0.98090 & 0.98030 & 0.98205 & 0.98180 & 0.98145 & 0.98230 \\
\hline \multicolumn{13}{|c|}{ Alcohol (\% vol.) } \\
\hline 0 & 15.11 & 15.11 & 15.11 & 12.97 & 12.97 & 12.97 & 14.40 & 14.40 & 14.40 & 14.15 & 14.15 & 14.15 \\
\hline 3 & 15.15 & 15.20 & 15.30 & 13.00 & 13.08 & 13.00 & 14.59 & 14.61 & 14.52 & 14.22 & 14.24 & 14.25 \\
\hline 6 & 15.05 & 15.48 & 14.80 & 13.13 & 13.30 & 12.32 & 14.79 & 15.07 & 14.39 & 13.99 & 14.50 & 13.77 \\
\hline 9 & 15.11 & 15.60 & 14.64 & 13.15 & 13.52 & 13.03 & 15.10 & 15.21 & 14.11 & 14.32 & 14.38 & 13.95 \\
\hline 12 & 14.87 & 15.65 & 14.70 & 12.59 & 13.45 & 12.98 & 14.81 & 15.38 & 13.76 & 14.02 & 14.33 & 13.56 \\
\hline \multicolumn{13}{|c|}{ Total dry extract (g/L) } \\
\hline $\mathbf{0}$ & 34.9 & 34.9 & 34.9 & 29.3 & 29.3 & 29.3 & 34.1 & 34.1 & 34.1 & 31.2 & 31.2 & 31.2 \\
\hline 3 & 35.1 & 35.1 & 35.9 & 28.9 & 29.3 & 29.3 & 35.1 & 34.2 & 34.1 & 31.0 & 31.0 & 31.6 \\
\hline 6 & 35.2 & 36.3 & 35.5 & 29.5 & 29.5 & 29.0 & 35.5 & 34.3 & 30.2 & 31.1 & 31.9 & 31.6 \\
\hline 9 & 35.2 & 36.7 & 35.5 & 29.3 & 30.5 & 29.7 & 35.4 & 34.2 & 30.3 & 31.1 & 31.0 & 31.4 \\
\hline 12 & 34.2 & 36.3 & 35.7 & 28.7 & 30.1 & 29.7 & 35.6 & 34.2 & 29.7 & 31.1 & 31.1 & 31.1 \\
\hline
\end{tabular}

Some physicochemical parameters of wine, like specific weight of wine, specific weight of distillate, alcohol percentage and dry extract were fairly stable during the period of storage of one year in different packaging (Table 1). Nevertheless, with statistical regression analysis, some statistically significant differences in these characteristics with time became visible (Table S1). Namely, specific weight of wine showed statistically significant difference over time in wines packed in multilayer and PET packaging. Specific weight of distillate and alcohol percentage were significantly different in wines packed in PET and multilayer packaging. Percentage of dry extract was significantly different in multilayer packaging after 6 months of storage. According to these results, glass bottles can be suggested as a good packaging for wine comparing to multilayer and PET containers, since no significant differences were shown in specific weight of wine, specific weight of distillate, alcohol percentage and dry extract in wines packed in glass bottles. However, it should be mentioned that all of wine parameters showed small differences. So, even though the statistical analysis pointed to some differences in physico-chemical parameters in wines packed in different packaging, those differences are not big. After creating regression models with the selected significant terms, the fitted values of the measured characteristics and their standard errors were also determined and shown (Table 2).

The amount of total and volatile acids in wines packed in different packaging during one year was similar (Figures 1 and 2). Again, with statistical regression analysis, some differences can be quantified (Table S1). Statistically significant differences were found for the change of volatile acid amount in time for wines packed in multilayer packaging (Table S1). Accordingly, it can be suggested that glass and PET packaging preserved total and volatile acids better than multilayer container. Fitted values of these parameters with their standard errors were shown in table 2 . 

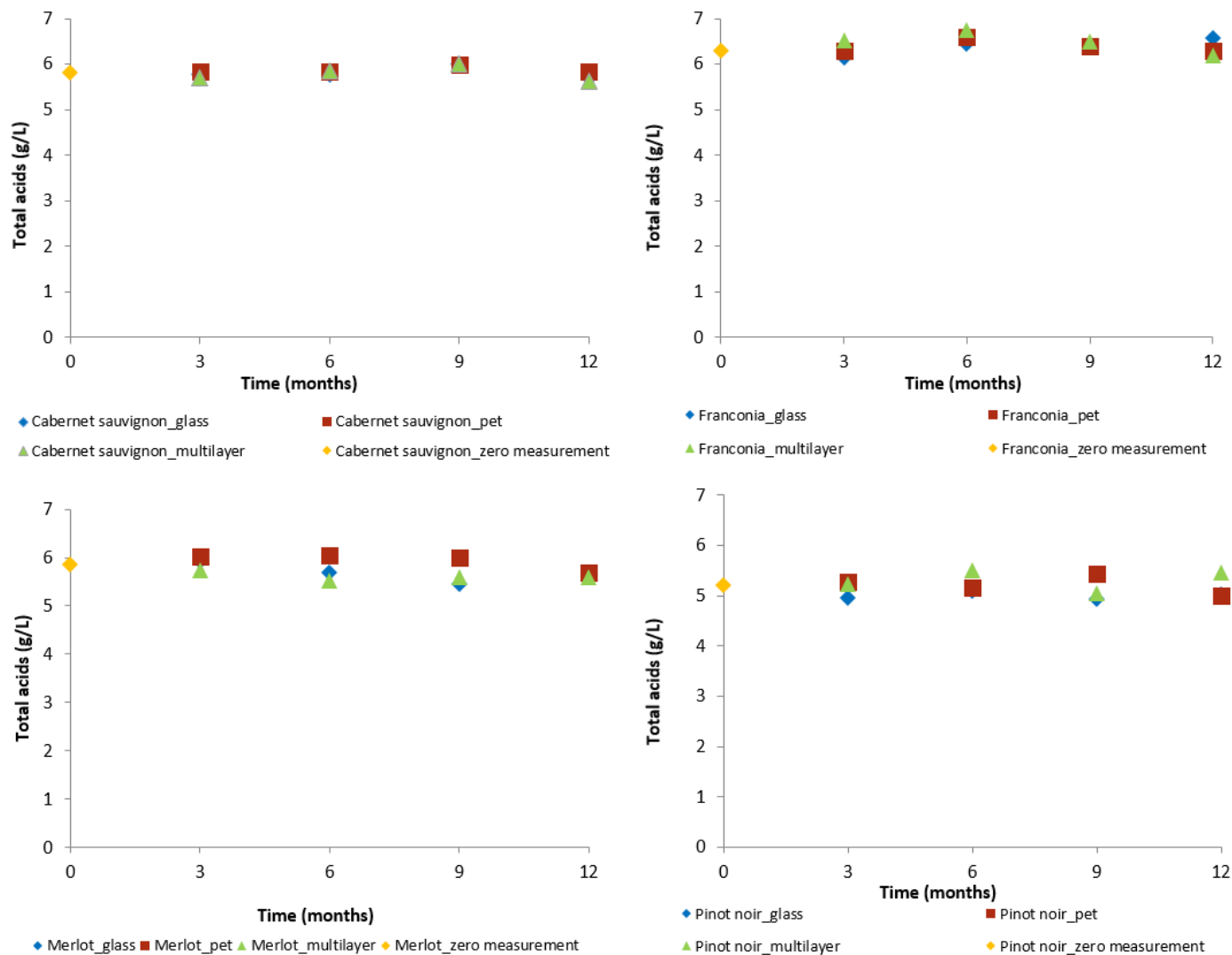

Figure 1 Total acids amount during storage of 12 months at $25^{\circ} \mathrm{C}$
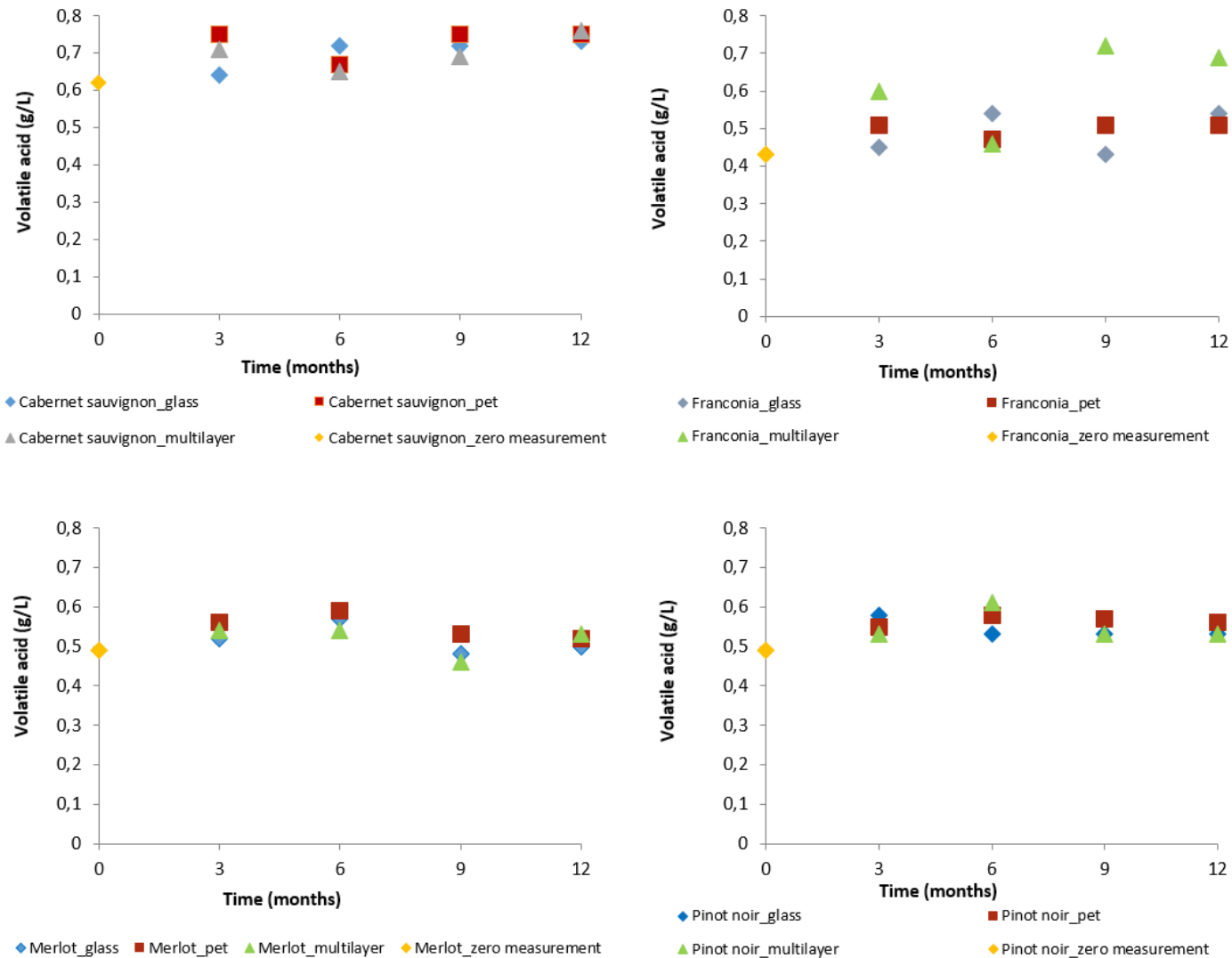

Figure 2 Volatile acids amount during storage of 12 months at $25^{\circ} \mathrm{C}$

Some differences can be seen in the free and total $\mathrm{SO}_{2}$ amount in dependence with time (Figures 3 and 4). Namely, the free and total $\mathrm{SO}_{2}$ were decreasing with time which is in accordance with earlier studies (Dimkou et al., 2011; Ghidossi et al., 2012). The central role played by $\mathrm{SO}_{2}$ is the consumption of oxygen. The decrease of $\mathrm{SO}_{2}$ concentration suggests that $\mathrm{O}_{2}$ is consumed and by that decreases the amount of $\mathrm{SO}_{2}$. Moreover, some differences in the free and total $\mathrm{SO}_{2}$ amount can also be seen in wines packed in different packaging. In the case of free $\mathrm{SO}_{2}$ (Figure 3), after three months of storage, wines packed in glass bottles contained the highest free $\mathrm{SO}_{2}$ amount, except Pinot noir. After 12 months of storage, higher free $\mathrm{SO}_{2}$ was found in wines packed in glass bottles (Cabernet sauvignon and Frankovka wine) or in PET bottles (Merlot, Pinot noir). In addition, free $\mathrm{SO}_{2}$ was the lowest in wines packed in multilayer containers. In the case of total $\mathrm{SO}_{2}$ 
(Figure 4), after three months of storage wines packed in glass bottles had the highest $\mathrm{SO}_{2}$ amount, and exception was again Pinot noir. After 12 months of storage, wines packed in glass and PET bottles had higher total $\mathrm{SO}_{2}$ amount (Merlot and Pinot noir) than wines packed in multilayer container, or the amount was similar (Cabernet sauvignon and Frankovka wine). According to these results, it can be suggested that glass and PET packaging protected the wine a little bit better than the multilayer container, due to the higher amount of $\mathrm{SO}_{2}$

Statistical regression conducted for free and total $\mathrm{SO}_{2}$ (Table S1) confirmed the suggested differences. Namely, the differences in the change of free $\mathrm{SO}_{2}$ and total $\mathrm{SO}_{2}$ amount over time were shown to be statistically significant. Moreover,
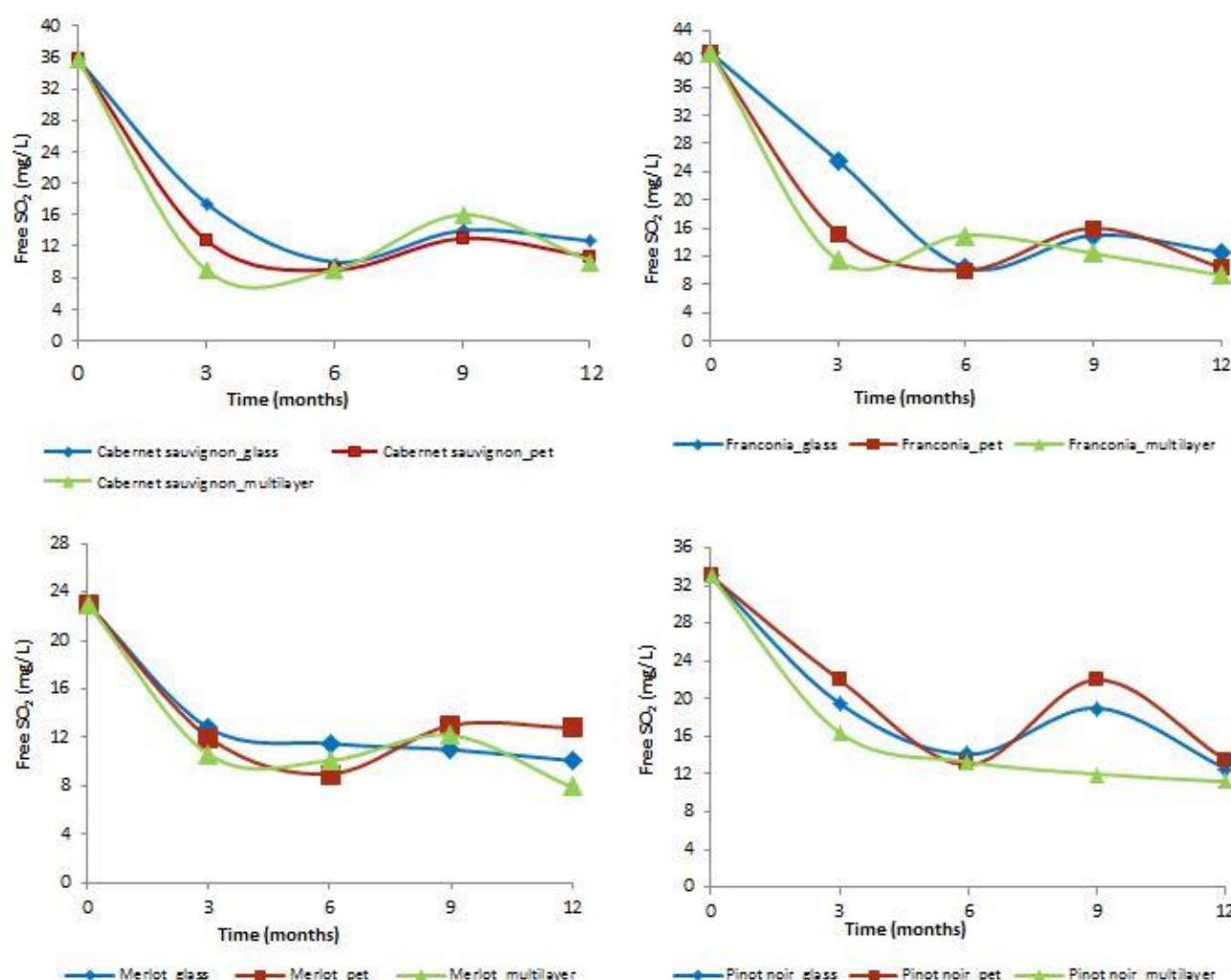

Figure 3 Free $\mathrm{SO}_{2}$ amount during storage of 12 months at $25^{\circ} \mathrm{C}$
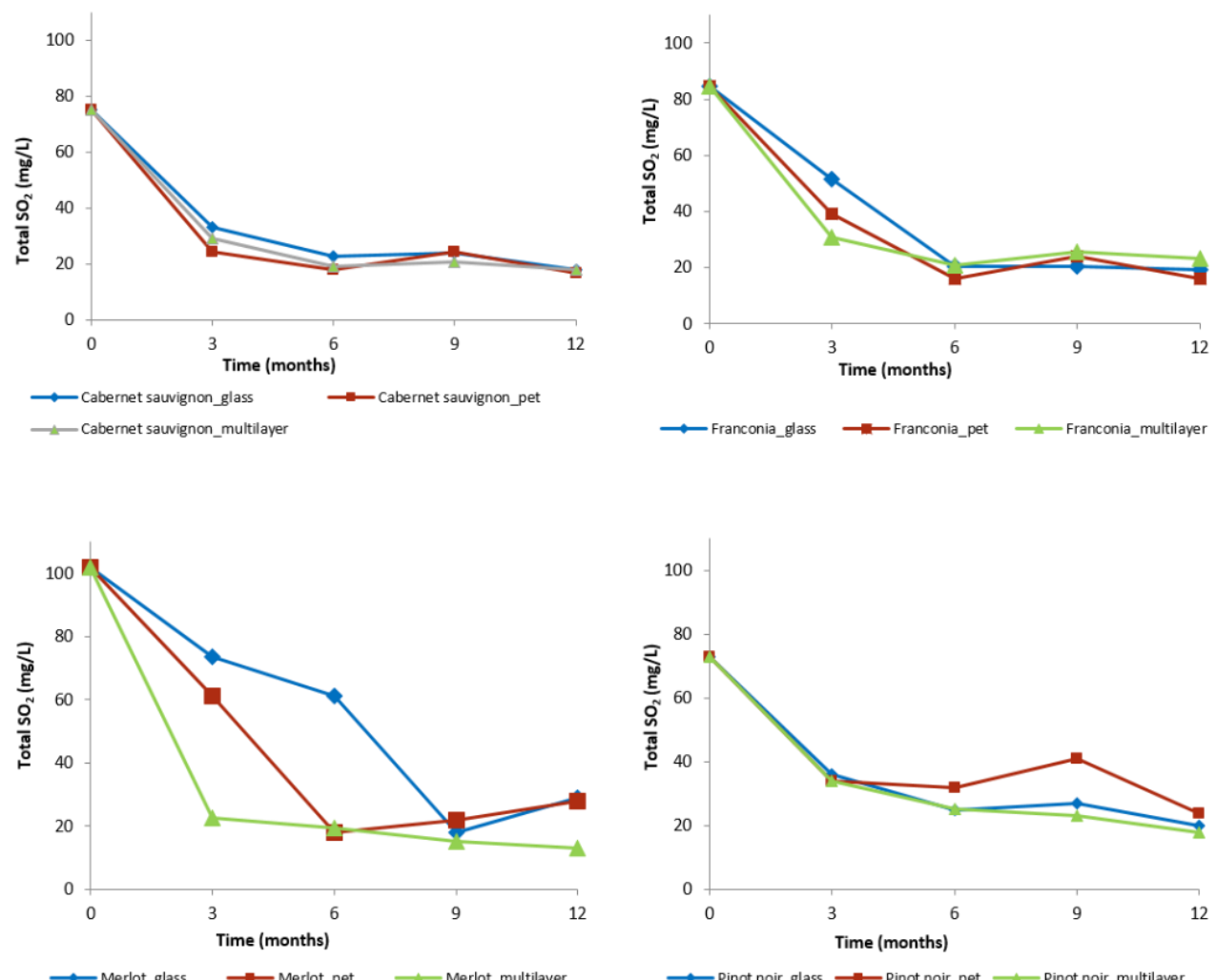

Figure 4 Total $\mathrm{SO}_{2}$ amount during storage of 12 months at $25^{\circ} \mathrm{C}$

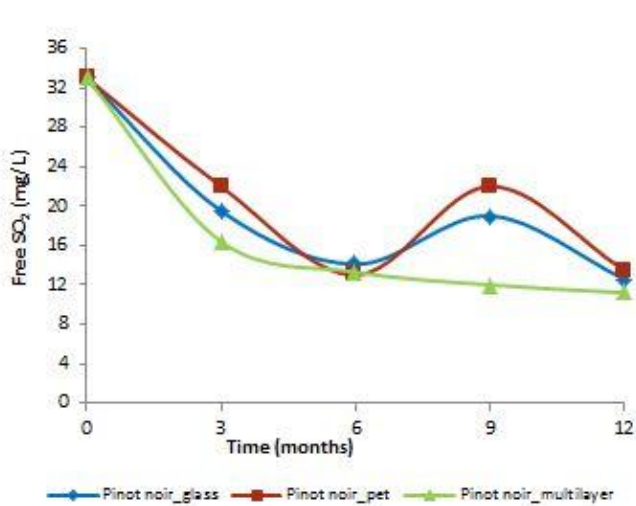

statistically significant differences were found in the changes of free and total $\mathrm{SO}_{2}$ amount in wines packed in different containers. Higher amount of free $\mathrm{SO}_{2}$ in the wine packed in glass after 3 months of storage was statistically significant. in glass and PET containers while lower total $\mathrm{SO}_{2}$ amount in wines in multilaye packaging were significant. The statistical analysis confirmed the observed differences in wines packed in different packaging materials. Fitted values of free and total $\mathrm{SO}_{2}$ with their standard errors are shown in table 2 . 
Table 2 Fited values of physico-chemical parameters and their standard errors (SE) obtained by using multiple regression and two variable interactions

\begin{tabular}{|c|c|c|c|c|c|c|c|c|c|c|c|c|}
\hline \multirow{2}{*}{$\begin{array}{l}\text { Time } \\
\text { (months) }\end{array}$} & \multicolumn{3}{|c|}{ Cabernet sauvignon } & \multicolumn{3}{|c|}{ Frankovka } & \multicolumn{3}{|c|}{ Merlot } & \multicolumn{3}{|c|}{ Pinot noir } \\
\hline & Glass & PET & $\begin{array}{c}\text { Multilaye } \\
\mathbf{r}\end{array}$ & Glass & PET & Multilayer & Glass & PET & Multilayer & Glass & PET & Multilayer \\
\hline \multicolumn{13}{|c|}{ Specific weight of wine } \\
\hline 0 & $\begin{array}{c}0.994228 \\
(0.000035)\end{array}$ & $\begin{array}{c}0.994228 \\
(0.000035)\end{array}$ & $\begin{array}{c}0.994228 \\
(0.000035)\end{array}$ & $\begin{array}{c}0.994228 \\
(0.000035)\end{array}$ & $\begin{array}{c}0.994228 \\
(0.000035)\end{array}$ & $\begin{array}{c}0.994228 \\
(0.000035)\end{array}$ & $\begin{array}{c}0.994690 \\
(0.000062)\end{array}$ & $\begin{array}{c}0.994690 \\
(0.000062)\end{array}$ & $\begin{array}{c}0.994690 \\
(0.000062)\end{array}$ & $\begin{array}{c}0.993765 \\
(0.000053)\end{array}$ & $\begin{array}{c}0.993765 \\
(0.000053)\end{array}$ & $\begin{array}{c}0.993765 \\
(0.000053)\end{array}$ \\
\hline 3 & $\begin{array}{c}0.994228 \\
(0.000035)\end{array}$ & $\begin{array}{c}0.994141 \\
(0.000032)\end{array}$ & $\begin{array}{c}0.994374 \\
(0.000032)\end{array}$ & $\begin{array}{c}0.994228 \\
(0.000035)\end{array}$ & $\begin{array}{c}0.994141 \\
(0.000032)\end{array}$ & $\begin{array}{c}0.994374 \\
(0.000032)\end{array}$ & $\begin{array}{c}0.994565 \\
(0.000051)\end{array}$ & $\begin{array}{c}0.994478 \\
(0.000048)\end{array}$ & $\begin{array}{c}0.994711 \\
(0.000051)\end{array}$ & $\begin{array}{c}0.993765 \\
(0.000053)\end{array}$ & $\begin{array}{c}0.993678 \\
(0.000049)\end{array}$ & $\begin{array}{c}0.993911 \\
(0.000048)\end{array}$ \\
\hline 6 & $\begin{array}{c}0.994228 \\
(0.000035)\end{array}$ & $\begin{array}{c}0.994054 \\
(0.000042)\end{array}$ & $\begin{array}{c}0.994520 \\
(0.000043)\end{array}$ & $\begin{array}{c}0.994228 \\
(0.000035)\end{array}$ & $\begin{array}{c}0.994054 \\
(0.000042)\end{array}$ & $\begin{array}{c}0.994520 \\
(0.000043)\end{array}$ & $\begin{array}{c}0.994439 \\
(0.000058)\end{array}$ & $\begin{array}{c}0.994265 \\
(0.000056)\end{array}$ & $\begin{array}{c}0.993346 \\
(0.000118)\end{array}$ & $\begin{array}{c}0.993765 \\
(0.000053)\end{array}$ & $\begin{array}{c}0.993591 \\
(0.000053)\end{array}$ & $\begin{array}{c}0.994057 \\
(0.000054)\end{array}$ \\
\hline 9 & $\begin{array}{c}0.994228 \\
(0.000035)\end{array}$ & $\begin{array}{c}0.993967 \\
(0.000058)\end{array}$ & $\begin{array}{c}0.994666 \\
(0.000062)\end{array}$ & $\begin{array}{c}0.994228 \\
(0.000035)\end{array}$ & $\begin{array}{c}0.993967 \\
(0.000058)\end{array}$ & $\begin{array}{c}0.994666 \\
(0.000062)\end{array}$ & $\begin{array}{c}0.994313 \\
(0.000079)\end{array}$ & $\begin{array}{c}0.994052 \\
(0.000080)\end{array}$ & $\begin{array}{c}0.993367 \\
(0.000111)\end{array}$ & $\begin{array}{c}0.993765 \\
(0.000053)\end{array}$ & $\begin{array}{c}0.993504 \\
(0.000065)\end{array}$ & $\begin{array}{c}0.994203 \\
(0.000068)\end{array}$ \\
\hline 12 & $\begin{array}{c}0.994228 \\
(0.000035) \\
\end{array}$ & $\begin{array}{c}0.993880 \\
(0.000077) \\
\end{array}$ & $\begin{array}{c}0.994812 \\
(0.000083) \\
\end{array}$ & $\begin{array}{c}0.994228 \\
(0.000035) \\
\end{array}$ & $\begin{array}{c}0.993880 \\
(0.000077) \\
\end{array}$ & $\begin{array}{c}0.994812 \\
(0.000083) \\
\end{array}$ & $\begin{array}{c}0.994187 \\
(0.000107) \\
\end{array}$ & $\begin{array}{c}0.993839 \\
(0.000110) \\
\end{array}$ & $\begin{array}{c}0.993387 \\
(0.000118) \\
\end{array}$ & $\begin{array}{c}0.993765 \\
(0.000053) \\
\end{array}$ & $\begin{array}{c}0.993417 \\
(0.000081) \\
\end{array}$ & $\begin{array}{c}0.994349 \\
(0.000085) \\
\end{array}$ \\
\hline \multicolumn{13}{|c|}{ Specific weight of distillate } \\
\hline 0 & $\begin{array}{c}0.980577 \\
(0.000048)\end{array}$ & $\begin{array}{c}0.980577 \\
(0.000081)\end{array}$ & $\begin{array}{c}0.980577 \\
(0.000081)\end{array}$ & $\begin{array}{c}0.982920 \\
(0.000048)\end{array}$ & $\begin{array}{c}0.982920 \\
(0.000081)\end{array}$ & $\begin{array}{c}0.982920 \\
(0.000081)\end{array}$ & $\begin{array}{c}0.981366 \\
(0.000035)\end{array}$ & $\begin{array}{c}0.981366 \\
(0.000074)\end{array}$ & $\begin{array}{c}0.981366 \\
(0.000074)\end{array}$ & $\begin{array}{c}0.981366 \\
(0.000035)\end{array}$ & $\begin{array}{c}0.981366 \\
(0.000074)\end{array}$ & $\begin{array}{c}0.981366 \\
(0.000074)\end{array}$ \\
\hline 3 & $\begin{array}{c}0.980577 \\
(0.000048)\end{array}$ & $\begin{array}{c}0.980552 \\
(0.000081)\end{array}$ & $\begin{array}{c}0.980602 \\
(0.000081)\end{array}$ & $\begin{array}{c}0.982920 \\
(0.000048)\end{array}$ & $\begin{array}{c}0.982895 \\
(0.000081)\end{array}$ & $\begin{array}{c}0.982945 \\
0.000081)\end{array}$ & $\begin{array}{c}0.981235 \\
(0.000038)\end{array}$ & $\begin{array}{c}0.981210 \\
(0.000076)\end{array}$ & $\begin{array}{c}0.981260 \\
(0.000076)\end{array}$ & $\begin{array}{c}0.981497 \\
(0.000037)\end{array}$ & $\begin{array}{c}0.981472 \\
(0.000075)\end{array}$ & $\begin{array}{c}0.981522 \\
(0.000075)\end{array}$ \\
\hline 6 & $\begin{array}{c}0.980577 \\
(0.000048)\end{array}$ & $\begin{array}{c}0.980147 \\
(0.000062)\end{array}$ & $\begin{array}{c}0.981007 \\
(0.000062)\end{array}$ & $\begin{array}{c}0.982920 \\
(0.000048)\end{array}$ & $\begin{array}{c}0.982490 \\
(0.000062)\end{array}$ & $\begin{array}{c}0.983350 \\
(0.000062)\end{array}$ & $\begin{array}{c}0.981104 \\
(0.000047)\end{array}$ & $\begin{array}{c}0.980674 \\
(0.000058)\end{array}$ & $\begin{array}{c}0.981534 \\
(0.000063)\end{array}$ & $\begin{array}{c}0.981628 \\
(0.000044)\end{array}$ & $\begin{array}{c}0.981197 \\
(0.000059)\end{array}$ & $\begin{array}{c}0.982058 \\
(0.000058)\end{array}$ \\
\hline 9 & $\begin{array}{c}0.980577 \\
(0.000048)\end{array}$ & $\begin{array}{c}0.980147 \\
(0.000062)\end{array}$ & $\begin{array}{c}0.981007 \\
(0.000062)\end{array}$ & $\begin{array}{c}0.982920 \\
(0.000048)\end{array}$ & $\begin{array}{c}0.982490 \\
(0.000062)\end{array}$ & $\begin{array}{c}0.983350 \\
(0.000062)\end{array}$ & $\begin{array}{c}0.980973 \\
(0.000057)\end{array}$ & $\begin{array}{c}0.980543 \\
(0.000067)\end{array}$ & $\begin{array}{c}0.981403 \\
(0.000072)\end{array}$ & $\begin{array}{c}0.981758 \\
(0.000054)\end{array}$ & $\begin{array}{c}0.981328 \\
(0.000068)\end{array}$ & $\begin{array}{c}0.982189 \\
(0.000066)\end{array}$ \\
\hline 12 & $\begin{array}{c}0.980577 \\
(0.000048) \\
\end{array}$ & $\begin{array}{c}0.980147 \\
(0.000062) \\
\end{array}$ & $\begin{array}{c}0.981007 \\
(0.000062) \\
\end{array}$ & $\begin{array}{c}0.982920 \\
(0.000048) \\
\end{array}$ & $\begin{array}{c}0.982490 \\
(0.000062) \\
\end{array}$ & $\begin{array}{c}0.983350 \\
(0.000062) \\
\end{array}$ & $\begin{array}{c}0.980842 \\
(0.000070) \\
\end{array}$ & $\begin{array}{c}0.980412 \\
(0.000077) \\
\end{array}$ & $\begin{array}{c}0.982050 \\
(0.000186) \\
\end{array}$ & $\begin{array}{c}0.981889 \\
(0.000066) \\
\end{array}$ & $\begin{array}{c}0.981459 \\
(0.000078) \\
\end{array}$ & $\begin{array}{c}0.982319 \\
(0.000075) \\
\end{array}$ \\
\hline$\overline{\text { Alcohol }(c}$ & vol.) & & & & & & & & & & & \\
\hline $\mathbf{0}$ & $\begin{array}{l}15.1253 \\
(0.0430)\end{array}$ & $\begin{array}{c}15.1253 \\
(0.0729)\end{array}$ & $\begin{array}{c}15.1253 \\
(0.0729)\end{array}$ & $\begin{array}{l}13.0307 \\
(0.0430)\end{array}$ & $\begin{array}{c}13.0307 \\
(0.0729)\end{array}$ & $\begin{array}{l}13.0307 \\
(0.0729)\end{array}$ & $\begin{array}{c}14.4089 \\
(0.0310)\end{array}$ & $\begin{array}{l}14.4089 \\
(0.0665)\end{array}$ & $\begin{array}{l}14.4089 \\
(0.0665)\end{array}$ & $\begin{array}{l}14.4089 \\
(0.0310)\end{array}$ & $\begin{array}{l}14.4089 \\
(0.0665)\end{array}$ & $\begin{array}{l}14.4089 \\
(0.0665)\end{array}$ \\
\hline 3 & $\begin{array}{l}15.1253 \\
(0.0430)\end{array}$ & $\begin{array}{c}15.1328 \\
(0.0729)\end{array}$ & $\begin{array}{c}15.1178 \\
(0.0729)\end{array}$ & $\begin{array}{l}13.0307 \\
(0.0430)\end{array}$ & $\begin{array}{c}13.0382 \\
(0.0729)\end{array}$ & $\begin{array}{l}13.0232 \\
(0.0729)\end{array}$ & $\begin{array}{l}14.5280 \\
(0.0343)\end{array}$ & $\begin{array}{l}14.5355 \\
(0.0681)\end{array}$ & $\begin{array}{c}14.5205 \\
(0.0681)\end{array}$ & $\begin{array}{l}14.2897 \\
(0.0329)\end{array}$ & $\begin{array}{c}14.2972 \\
(0.0674)\end{array}$ & $\begin{array}{c}14.2822 \\
(0.0674)\end{array}$ \\
\hline 6 & $\begin{array}{l}15.1253 \\
(0.0430)\end{array}$ & $\begin{array}{l}15.5051 \\
(0.0553)\end{array}$ & $\begin{array}{r}14.7456 \\
(0.0553)\end{array}$ & $\begin{array}{l}13.0307 \\
(0.0430)\end{array}$ & $\begin{array}{r}13.4104 \\
(0.0553)\end{array}$ & $\begin{array}{l}12.6509 \\
(0.0553)\end{array}$ & $\begin{array}{l}14.6471 \\
(0.0417)\end{array}$ & $\begin{array}{l}15.0269 \\
(0.0523)\end{array}$ & $\begin{array}{l}14.2673 \\
(0.0563)\end{array}$ & $\begin{array}{l}14.1706 \\
(0.0393)\end{array}$ & $\begin{array}{l}14.5504 \\
(0.0528)\end{array}$ & $\begin{array}{l}13.7908 \\
(0.0522)\end{array}$ \\
\hline 9 & $\begin{array}{l}15.1253 \\
(0.0430)\end{array}$ & $\begin{array}{l}15.5051 \\
(0.0553)\end{array}$ & $\begin{array}{l}14.7456 \\
(0.0553)\end{array}$ & $\begin{array}{l}13.0307 \\
(0.0430)\end{array}$ & $\begin{array}{l}13.4104 \\
(0.0553)\end{array}$ & $\begin{array}{l}12.6509 \\
(0.0553)\end{array}$ & $\begin{array}{l}14.7662 \\
(0.0514)\end{array}$ & $\begin{array}{l}15.1460 \\
(0.0598)\end{array}$ & $\begin{array}{c}14.3865 \\
(0.0642)\end{array}$ & $\begin{array}{l}14.0515 \\
(0.0484)\end{array}$ & $\begin{array}{l}14.4312 \\
(0.0604)\end{array}$ & $\begin{array}{l}13.6717 \\
(0.0589)\end{array}$ \\
\hline 12 & $\begin{array}{l}15.1253 \\
(0.0430) \\
\end{array}$ & $\begin{array}{r}15.5051 \\
(0.0553) \\
\end{array}$ & $\begin{array}{r}14.7456 \\
(0.0553) \\
\end{array}$ & $\begin{array}{r}13.0307 \\
(0.0430) \\
\end{array}$ & $\begin{array}{r}13.4104 \\
(0.0553) \\
\end{array}$ & $\begin{array}{r}12.6509 \\
(0.0553) \\
\end{array}$ & $\begin{array}{r}14.8853 \\
(0.0623) \\
\end{array}$ & $\begin{array}{l}15.2651 \\
(0.0690) \\
\end{array}$ & $\begin{array}{r}13.7600 \\
(0.1664) \\
\end{array}$ & $\begin{array}{r}13.9324 \\
(0.0591) \\
\end{array}$ & $\begin{array}{r}14.3121 \\
(0.0697) \\
\end{array}$ & $\begin{array}{r}13.5526 \\
(0.0674) \\
\end{array}$ \\
\hline Total dry & xtract $(g / L)$ & & & & & & & & & & & \\
\hline $\mathbf{0}$ & $\begin{array}{l}35.427 \\
(0.132)\end{array}$ & $\begin{array}{l}35.427 \\
(0.132)\end{array}$ & $\begin{array}{l}35.427 \\
(0.132)\end{array}$ & $\begin{array}{l}29.427 \\
(0.132)\end{array}$ & $\begin{array}{l}29.427 \\
(0.132)\end{array}$ & $\begin{array}{l}29.427 \\
(0.132)\end{array}$ & $\begin{array}{l}34.575 \\
(0.148)\end{array}$ & $\begin{array}{l}34.575 \\
(0.148)\end{array}$ & $\begin{array}{l}34.575 \\
(0.148)\end{array}$ & $\begin{array}{l}31.240 \\
(0.132)\end{array}$ & $\begin{array}{l}31.240 \\
(0.132)\end{array}$ & $\begin{array}{l}31.240 \\
(0.132)\end{array}$ \\
\hline 3 & $\begin{array}{l}35.427 \\
(0.132)\end{array}$ & $\begin{array}{l}35.427 \\
(0.132)\end{array}$ & $\begin{array}{l}35.427 \\
(0.132)\end{array}$ & $\begin{array}{l}29.427 \\
(0.132)\end{array}$ & $\begin{array}{l}29.427 \\
(0.132)\end{array}$ & $\begin{array}{l}29.427 \\
(0.132)\end{array}$ & $\begin{array}{l}34.575 \\
(0.148)\end{array}$ & $\begin{array}{l}34.575 \\
(0.148)\end{array}$ & $\begin{array}{l}34.575 \\
(0.148)\end{array}$ & $\begin{array}{l}31.240 \\
(0.132)\end{array}$ & $\begin{array}{l}31.240 \\
(0.132)\end{array}$ & $\begin{array}{l}31.240 \\
(0.132)\end{array}$ \\
\hline 6 & $\begin{array}{l}35.427 \\
(0.132)\end{array}$ & $\begin{array}{l}35.427 \\
(0.132)\end{array}$ & $\begin{array}{l}35.427 \\
(0.132)\end{array}$ & $\begin{array}{l}29.427 \\
(0.132)\end{array}$ & $\begin{array}{l}29.427 \\
(0.132)\end{array}$ & $\begin{array}{l}29.427 \\
(0.132)\end{array}$ & $\begin{array}{l}34.575 \\
(0.148)\end{array}$ & $\begin{array}{l}34.575 \\
(0.148)\end{array}$ & $\begin{array}{l}30.067 \\
(0.296)\end{array}$ & $\begin{array}{l}31.240 \\
(0.132)\end{array}$ & $\begin{array}{l}31.240 \\
(0.132)\end{array}$ & $\begin{array}{l}31.240 \\
(0.132)\end{array}$ \\
\hline 9 & $\begin{array}{l}35.427 \\
(0.132)\end{array}$ & $\begin{array}{l}35.427 \\
(0.132)\end{array}$ & $\begin{array}{l}35.427 \\
(0.132)\end{array}$ & $\begin{array}{l}29.427 \\
(0.132)\end{array}$ & $\begin{array}{l}29.427 \\
(0.132)\end{array}$ & $\begin{array}{l}29.427 \\
(0.132)\end{array}$ & $\begin{array}{l}34.575 \\
(0.148)\end{array}$ & $\begin{array}{l}34.575 \\
(0.148)\end{array}$ & $\begin{array}{l}30.067 \\
(0.296)\end{array}$ & $\begin{array}{l}31.240 \\
(0.132)\end{array}$ & $\begin{array}{l}31.240 \\
(0.132)\end{array}$ & $\begin{array}{l}31.240 \\
(0.132)\end{array}$ \\
\hline 12 & $\begin{array}{r}35.427 \\
(0.132) \\
\end{array}$ & $\begin{array}{l}35.427 \\
(0.132) \\
\end{array}$ & $\begin{array}{l}35.427 \\
(0.132) \\
\end{array}$ & $\begin{array}{l}29.427 \\
(0.132) \\
\end{array}$ & $\begin{array}{l}29.427 \\
(0.132) \\
\end{array}$ & $\begin{array}{r}29.427 \\
(0.132) \\
\end{array}$ & $\begin{array}{l}34.575 \\
(0.148) \\
\end{array}$ & $\begin{array}{l}34.575 \\
(0.148) \\
\end{array}$ & $\begin{array}{l}30.067 \\
(0.296) \\
\end{array}$ & $\begin{array}{l}31.240 \\
(0.132) \\
\end{array}$ & $\begin{array}{l}31.240 \\
(0.132) \\
\end{array}$ & $\begin{array}{l}31.240 \\
(0.132) \\
\end{array}$ \\
\hline Total acid & $\mathrm{g} / \mathrm{L})$ & & & & & & & & & & & \\
\hline O & $\begin{array}{c}5.7937 \\
(0.0212)\end{array}$ & $\begin{array}{c}5.7937 \\
(0.0212)\end{array}$ & $\begin{array}{c}5.7937 \\
(0.0212)\end{array}$ & $\begin{array}{c}6.4121 \\
(0.0406)\end{array}$ & $\begin{array}{c}6.4121 \\
(0.0406)\end{array}$ & $\begin{array}{c}6.4121 \\
(0.0406)\end{array}$ & $\begin{array}{c}5.7754 \\
(0.0406)\end{array}$ & $\begin{array}{c}5.7754 \\
(0.0406)\end{array}$ & $\begin{array}{c}5.7754 \\
(0.0406)\end{array}$ & $\begin{array}{c}5.1934 \\
(0.0406)\end{array}$ & $\begin{array}{c}5.1934 \\
(0.0406)\end{array}$ & $\begin{array}{c}5.1934 \\
(0.0406)\end{array}$ \\
\hline 3 & $\begin{array}{c}5.7937 \\
(0.0212)\end{array}$ & $\begin{array}{c}5.7937 \\
(0.0212)\end{array}$ & $\begin{array}{c}5.7937 \\
(0.0212)\end{array}$ & $\begin{array}{c}6.4121 \\
(0.0406)\end{array}$ & $\begin{array}{c}6.4121 \\
(0.0406)\end{array}$ & $\begin{array}{c}6.4121 \\
(0.0406)\end{array}$ & $\begin{array}{c}5.7754 \\
(0.0406)\end{array}$ & $\begin{array}{c}5.7754 \\
(0.0406)\end{array}$ & $\begin{array}{c}5.7754 \\
(0.0406)\end{array}$ & $\begin{array}{c}5.1934 \\
(0.0406)\end{array}$ & $\begin{array}{c}5.1934 \\
(0.0406)\end{array}$ & $\begin{array}{c}5.1934 \\
(0.0406)\end{array}$ \\
\hline 6 & $\begin{array}{c}5.7937 \\
(0.0212)\end{array}$ & $\begin{array}{c}5.7937 \\
(0.0212)\end{array}$ & $\begin{array}{c}5.7937 \\
(0.0212)\end{array}$ & $\begin{array}{c}6.4121 \\
(0.0406)\end{array}$ & $\begin{array}{c}6.4121 \\
(0.0406)\end{array}$ & $\begin{array}{c}6.4121 \\
(0.0406)\end{array}$ & $\begin{array}{c}5.7754 \\
(0.0406)\end{array}$ & $\begin{array}{c}5.7754 \\
(0.0406)\end{array}$ & $\begin{array}{c}5.7754 \\
(0.0406)\end{array}$ & $\begin{array}{c}5.1934 \\
(0.0406)\end{array}$ & $\begin{array}{c}5.1934 \\
(0.0406)\end{array}$ & $\begin{array}{c}5.1934 \\
(0.0406)\end{array}$ \\
\hline 9 & $\begin{array}{c}5.7937 \\
(0.0212)\end{array}$ & $\begin{array}{c}5.7937 \\
(0.0212)\end{array}$ & $\begin{array}{c}5.7937 \\
(0.0212)\end{array}$ & $\begin{array}{c}6.4121 \\
(0.0406)\end{array}$ & $\begin{array}{c}6.4121 \\
(0.0406)\end{array}$ & $\begin{array}{c}6.4121 \\
(0.0406)\end{array}$ & $\begin{array}{c}5.7754 \\
(0.0406)\end{array}$ & $\begin{array}{c}5.7754 \\
(0.0406)\end{array}$ & $\begin{array}{c}5.7754 \\
(0.0406)\end{array}$ & $\begin{array}{c}5.1934 \\
(0.0406)\end{array}$ & $\begin{array}{c}5.1934 \\
(0.0406)\end{array}$ & $\begin{array}{c}5.1934 \\
(0.0406)\end{array}$ \\
\hline 12 & $\begin{array}{c}5.7937 \\
(0.0212) \\
\end{array}$ & $\begin{array}{c}5.7937 \\
(0.0212) \\
\end{array}$ & $\begin{array}{c}5.7937 \\
(0.0212) \\
\end{array}$ & $\begin{array}{c}6.4121 \\
(0.0406) \\
\end{array}$ & $\begin{array}{c}6.4121 \\
(0.0406) \\
\end{array}$ & $\begin{array}{c}6.4121 \\
(0.0406) \\
\end{array}$ & $\begin{array}{c}5.7754 \\
(0.0406) \\
\end{array}$ & $\begin{array}{c}5.7754 \\
(0.0406) \\
\end{array}$ & $\begin{array}{c}5.7754 \\
(0.0406) \\
\end{array}$ & $\begin{array}{c}5.1934 \\
(0.0406) \\
\end{array}$ & $\begin{array}{c}5.1934 \\
(0.0406) \\
\end{array}$ & $\begin{array}{c}5.1934 \\
(0.0406) \\
\end{array}$ \\
\hline Volatile a & $d(g / L)$ & & & & & & & & & & & \\
\hline $\mathbf{0}$ & $\begin{array}{c}0.6714 \\
(0.0128)\end{array}$ & $\begin{array}{c}0.6714 \\
(0.0128)\end{array}$ & $\begin{array}{c}0.6714 \\
(0.0128)\end{array}$ & $\begin{array}{c}0.4360 \\
(0.0214)\end{array}$ & $\begin{array}{c}0.4360 \\
(0.0214)\end{array}$ & $\begin{array}{c}0.4360 \\
(0.0214)\end{array}$ & $\begin{array}{c}0.5244 \\
(0.0094)\end{array}$ & $\begin{array}{c}0.5244 \\
(0.0094)\end{array}$ & $\begin{array}{c}0.5244 \\
(0.0094)\end{array}$ & $\begin{array}{c}0.5244 \\
(0.0094)\end{array}$ & $\begin{array}{c}0.5244 \\
(0.0094)\end{array}$ & $\begin{array}{c}0.5244 \\
(0.0094)\end{array}$ \\
\hline 3 & $\begin{array}{c}0.6714 \\
(0.0128)\end{array}$ & $\begin{array}{c}0.6714 \\
(0.0128)\end{array}$ & $\begin{array}{c}0.6903 \\
(0.0125)\end{array}$ & $\begin{array}{c}0.4690 \\
(0.0153)\end{array}$ & $\begin{array}{c}0.4690 \\
(0.0153)\end{array}$ & $\begin{array}{c}0.4879 \\
(0.0155)\end{array}$ & $\begin{array}{c}0.5244 \\
(0.0094)\end{array}$ & $\begin{array}{c}0.5244 \\
(0.0094)\end{array}$ & $\begin{array}{c}0.5433 \\
(0.0089)\end{array}$ & $\begin{array}{c}0.5244 \\
(0.0094)\end{array}$ & $\begin{array}{c}0.5244 \\
(0.0094)\end{array}$ & $\begin{array}{c}0.5433 \\
(0.0089)\end{array}$ \\
\hline 6 & $\begin{array}{c}0.6714 \\
(0.0128)\end{array}$ & $\begin{array}{c}0.6714 \\
(0.0128)\end{array}$ & $\begin{array}{c}0.7092 \\
(0.0141)\end{array}$ & $\begin{array}{c}0.5021 \\
(0.0128)\end{array}$ & $\begin{array}{c}0.5021 \\
(0.0128)\end{array}$ & $\begin{array}{c}0.5399 \\
(0.0141)\end{array}$ & $\begin{array}{c}0.5244 \\
(0.0094)\end{array}$ & $\begin{array}{c}0.5244 \\
(0.0094)\end{array}$ & $\begin{array}{c}0.5622 \\
(0.0110)\end{array}$ & $\begin{array}{c}0.5244 \\
(0.0094)\end{array}$ & $\begin{array}{c}0.5244 \\
(0.0094)\end{array}$ & $\begin{array}{c}0.5622 \\
(0.0110)\end{array}$ \\
\hline 9 & $\begin{array}{c}0.6714 \\
(0.0128)\end{array}$ & $\begin{array}{c}0.6714 \\
(0.0128)\end{array}$ & $\begin{array}{c}0.7281 \\
(0.0171)\end{array}$ & $\begin{array}{c}0.5351 \\
(0.0160)\end{array}$ & $\begin{array}{c}0.5351 \\
(0.0160)\end{array}$ & $\begin{array}{c}0.5918 \\
(0.0182)\end{array}$ & $\begin{array}{c}0.5244 \\
(0.0094)\end{array}$ & $\begin{array}{c}0.5244 \\
(0.0094)\end{array}$ & $\begin{array}{c}0.5811 \\
(0.0147)\end{array}$ & $\begin{array}{c}0.5244 \\
(0.0094)\end{array}$ & $\begin{array}{c}0.5244 \\
(0.0094)\end{array}$ & $\begin{array}{c}0.5811 \\
(0.0147)\end{array}$ \\
\hline 12 & $\begin{array}{c}0.6714 \\
(0.0128) \\
\end{array}$ & $\begin{array}{c}0.6714 \\
(0.0128) \\
\end{array}$ & $\begin{array}{c}0.7470 \\
(0.0209) \\
\end{array}$ & $\begin{array}{c}0.5681 \\
(0.0225) \\
\end{array}$ & $\begin{array}{c}0.5681 \\
(0.0225) \\
\end{array}$ & $\begin{array}{c}0.6437 \\
(0.0253) \\
\end{array}$ & $\begin{array}{c}0.5244 \\
(0.0094) \\
\end{array}$ & $\begin{array}{c}0.5244 \\
(0.0094) \\
\end{array}$ & $\begin{array}{c}0.6000 \\
(0.0190) \\
\end{array}$ & $\begin{array}{c}0.5244 \\
(0.0094) \\
\end{array}$ & $\begin{array}{c}0.5244 \\
(0.0094) \\
\end{array}$ & $\begin{array}{c}0.6000 \\
(0.0190) \\
\end{array}$ \\
\hline Free $\mathrm{SO}_{2}$ & $\mathrm{gg} / \mathrm{L})$ & & & & & & & & & & & \\
\hline $\mathbf{0}$ & $\begin{array}{l}33.18 \\
(0.83)\end{array}$ & $\begin{array}{l}12.80 \\
(0.42)\end{array}$ & $\begin{array}{l}12.80 \\
(0.42)\end{array}$ & $\begin{array}{l}25.60 \\
(2.86)\end{array}$ & $\begin{array}{l}10.93 \\
(0.52)\end{array}$ & $\begin{array}{l}10.93 \\
(0.52)\end{array}$ & $10.88(0.68)$ & $\begin{array}{l}10.88 \\
(0.68)\end{array}$ & $\begin{array}{l}10.88 \\
(0.68)\end{array}$ & $\begin{array}{l}16.58 \\
(0.68)\end{array}$ & $\begin{array}{l}32.29 \\
(1.15)\end{array}$ & $\begin{array}{l}32.29 \\
(1.15)\end{array}$ \\
\hline 3 & $\begin{array}{l}12.80 \\
(0.42)\end{array}$ & $\begin{array}{l}12.80 \\
(0.42)\end{array}$ & $\begin{array}{l}12.80 \\
(0.42)\end{array}$ & $\begin{array}{l}10.93 \\
(0.52)\end{array}$ & $\begin{array}{l}10.93 \\
(0.52)\end{array}$ & $\begin{array}{l}10.93 \\
(0.52)\end{array}$ & $10.88(0.68)$ & $\begin{array}{l}10.88 \\
(0.68)\end{array}$ & $\begin{array}{l}10.88 \\
(0.68)\end{array}$ & $\begin{array}{l}16.58 \\
(0.68)\end{array}$ & $\begin{array}{l}16.58 \\
(0.68)\end{array}$ & $\begin{array}{l}16.58 \\
(0.68)\end{array}$ \\
\hline 6 & $\begin{array}{l}12.80 \\
(0.42)\end{array}$ & $\begin{array}{l}12.80 \\
(0.42)\end{array}$ & $\begin{array}{l}12.80 \\
(0.42)\end{array}$ & $\begin{array}{l}10.93 \\
(0.52)\end{array}$ & $\begin{array}{l}10.93 \\
(0.52)\end{array}$ & $\begin{array}{l}10.93 \\
(0.52)\end{array}$ & $10.88(0.68)$ & $\begin{array}{l}10.88 \\
(0.68)\end{array}$ & $\begin{array}{l}10.88 \\
(0.68)\end{array}$ & $\begin{array}{l}16.58 \\
(0.68)\end{array}$ & $\begin{array}{l}16.58 \\
(0.68)\end{array}$ & $\begin{array}{l}16.58 \\
(0.68)\end{array}$ \\
\hline 9 & $\begin{array}{l}12.80 \\
(0.42)\end{array}$ & $\begin{array}{l}12.80 \\
(0.42)\end{array}$ & $\begin{array}{l}12.80 \\
(0.42)\end{array}$ & $\begin{array}{l}10.93 \\
(0.52)\end{array}$ & $\begin{array}{l}10.93 \\
(0.52)\end{array}$ & $\begin{array}{l}10.93 \\
(0.52)\end{array}$ & $10.88(0.68)$ & $\begin{array}{l}10.88 \\
(0.68)\end{array}$ & $\begin{array}{l}10.88 \\
(0.68)\end{array}$ & $\begin{array}{l}16.58 \\
(0.68)\end{array}$ & $\begin{array}{l}16.58 \\
(0.68)\end{array}$ & $\begin{array}{l}16.58 \\
(0.68)\end{array}$ \\
\hline 12 & $\begin{array}{l}33.18 \\
(0.83)\end{array}$ & $\begin{array}{l}33.18 \\
(0.83)\end{array}$ & $\begin{array}{l}40.64 \\
(1.47)\end{array}$ & $\begin{array}{l}40.64 \\
(1.47)\end{array}$ & $\begin{array}{l}40.64 \\
(1.47)\end{array}$ & $\begin{array}{l}26.59 \\
(1.15)\end{array}$ & $26.59(1.15)$ & $\begin{array}{l}26.59 \\
(1.15)\end{array}$ & $\begin{array}{l}32.29 \\
(1.15)\end{array}$ & $\begin{array}{l}32.29 \\
(1.15)\end{array}$ & $\begin{array}{l}16.58 \\
(0.68)\end{array}$ & $\begin{array}{l}16.58 \\
(0.68)\end{array}$ \\
\hline Total $\mathrm{SO}_{2}$ & $\mathrm{mg} / \mathrm{L})$ & & & & & & & & & & & \\
\hline 0 & $\begin{array}{r}78.30 \\
(1.54)\end{array}$ & $\begin{array}{l}78.30 \\
(1.54)\end{array}$ & $\begin{array}{l}78.30 \\
(1.54)\end{array}$ & $\begin{array}{l}85.50 \\
(1.25)\end{array}$ & $\begin{array}{l}85.50 \\
(1.25)\end{array}$ & $\begin{array}{l}85.50 \\
(1.25)\end{array}$ & $100.00(2.12)$ & $\begin{array}{l}100.00 \\
(2.12)\end{array}$ & $\begin{array}{l}100.00 \\
(2.12)\end{array}$ & $\begin{array}{l}71.00 \\
(2.12)\end{array}$ & $\begin{array}{l}71.00 \\
(2.12)\end{array}$ & $\begin{array}{l}71.00 \\
(2.12)\end{array}$ \\
\hline 3 & 28.15 & 28.15 & 28.15 & 49.66 & 49.66 & 30.70 & $65.73(2.67)$ & 65.73 & 22.60 & 33.59 & 33.59 & 33.59 \\
\hline & $(2.37)$ & $(2.37)$ & $(2.37)$ & $(1.59)$ & $(1.59)$ & $(4.19)$ & & $(2.67)$ & $(4.19)$ & $(2.26)$ & $(2.26)$ & $(2.26)$ \\
\hline 6 & $\begin{array}{l}19.51 \\
(1.31)\end{array}$ & $\begin{array}{l}19.51 \\
(1.31)\end{array}$ & $\begin{array}{l}19.51 \\
(1.31)\end{array}$ & $\begin{array}{l}21.94 \\
(0.82)\end{array}$ & $\begin{array}{l}21.94 \\
(0.82)\end{array}$ & $\begin{array}{l}21.94 \\
(0.82)\end{array}$ & $61.00(4.19)$ & $\begin{array}{l}19.93 \\
(1.33)\end{array}$ & $\begin{array}{l}19.93 \\
(1.33)\end{array}$ & $\begin{array}{l}23.94 \\
(1.33)\end{array}$ & $\begin{array}{l}23.94 \\
(1.33)\end{array}$ & $\begin{array}{l}23.94 \\
(1.33)\end{array}$ \\
\hline 9 & 19.51 & 19.51 & 19.51 & 21.94 & 21.94 & 21.94 & $19.93(1.33)$ & 19.93 & 19.93 & 23.94 & 41.00 & 23.94 \\
\hline & $(1.31)$ & $(1.31)$ & $(1.31)$ & $(0.82)$ & $(0.82)$ & $(0.82)$ & & $(1.33)$ & $(1.33)$ & $(1.33)$ & (4.19) & $(1.33)$ \\
\hline 12 & $\begin{array}{l}19.51 \\
(1.31) \\
\end{array}$ & $\begin{array}{l}19.51 \\
(1.31) \\
\end{array}$ & $\begin{array}{l}19.51 \\
(1.31) \\
\end{array}$ & $\begin{array}{l}21.94 \\
(0.82) \\
\end{array}$ & $\begin{array}{l}21.94 \\
(0.82) \\
\end{array}$ & $\begin{array}{l}21.94 \\
(0.82) \\
\end{array}$ & $19.93(1.33)$ & $\begin{array}{r}19.93 \\
(1.33) \\
\end{array}$ & $\begin{array}{l}19.93 \\
(1.33) \\
\end{array}$ & $\begin{array}{l}23.94 \\
(1.33) \\
\end{array}$ & $\begin{array}{l}23.94 \\
(1.33) \\
\end{array}$ & $\begin{array}{l}23.94 \\
(1.33) \\
\end{array}$ \\
\hline Oxygen (n & & & & & & & & & & & & \\
\hline o & $\begin{array}{c}9.016 \\
(0.196)\end{array}$ & $\begin{array}{c}8.501 \\
(0.178)\end{array}$ & $\begin{array}{c}8.501 \\
(0.178)\end{array}$ & $\begin{array}{c}9.016 \\
(0.196)\end{array}$ & $\begin{array}{c}8.501 \\
(0.178)\end{array}$ & $\begin{array}{c}8.501 \\
(0.178)\end{array}$ & $6.865(0.311)$ & $\begin{array}{c}6.350 \\
(0.300)\end{array}$ & $\begin{array}{c}6.350 \\
(0.300)\end{array}$ & $\begin{array}{c}9.437 \\
(0.218)\end{array}$ & $\begin{array}{c}8.922 \\
(0.202)\end{array}$ & $\begin{array}{c}8.922 \\
(0.202)\end{array}$ \\
\hline 3 & $\begin{array}{c}2.326 \\
(0.124)\end{array}$ & $\begin{array}{c}1.811 \\
(0.092)\end{array}$ & $\begin{array}{c}1.811 \\
(0.092)\end{array}$ & $\begin{array}{c}2.326 \\
(0.124)\end{array}$ & $\begin{array}{c}1.811 \\
(0.092)\end{array}$ & $\begin{array}{c}1.811 \\
(0.092)\end{array}$ & $2.337(0.166)$ & $\begin{array}{c}1.822 \\
(0.144)\end{array}$ & $\begin{array}{c}1.822 \\
(0.144)\end{array}$ & $\begin{array}{c}2.747 \\
(0.157)\end{array}$ & $\begin{array}{c}2.232 \\
(0.133)\end{array}$ & $\begin{array}{c}2.232 \\
(0.133)\end{array}$ \\
\hline
\end{tabular}




\begin{tabular}{|c|c|c|c|c|c|c|c|c|c|c|c|c|}
\hline 6 & $\begin{array}{c}2.326 \\
(0.124)\end{array}$ & $\begin{array}{c}1.811 \\
(0.092)\end{array}$ & $\begin{array}{c}1.811 \\
(0.092)\end{array}$ & $\begin{array}{c}2.326 \\
(0.124)\end{array}$ & $\begin{array}{c}1.811 \\
(0.092)\end{array}$ & $\begin{array}{c}1.811 \\
(0.092)\end{array}$ & $2.337(0.166)$ & $\begin{array}{c}1.822 \\
(0.144)\end{array}$ & $\begin{array}{c}1.822 \\
(0.144)\end{array}$ & $\begin{array}{c}2.747 \\
(0.157)\end{array}$ & $\begin{array}{c}2.232 \\
(0.133)\end{array}$ & $\begin{array}{c}2.232 \\
(0.133)\end{array}$ \\
\hline 9 & 2.326 & 1.811 & 1.811 & 2.326 & 1.811 & 1.811 & $2.337(0.166)$ & 1.822 & 1.822 & 2.747 & 2.232 & 2.232 \\
\hline & $(0.124)$ & $(0.092)$ & $(0.092)$ & $(0.124)$ & $(0.092)$ & (0.092) & & (0.144) & $(0.144)$ & $(0.157)$ & $(0.133)$ & $(0.133)$ \\
\hline 12 & $\begin{array}{c}2.326 \\
(0.124)\end{array}$ & $\begin{array}{c}1.811 \\
(0.092)\end{array}$ & $\begin{array}{c}1.811 \\
(0.092)\end{array}$ & $\begin{array}{c}2.326 \\
(0.124)\end{array}$ & $\begin{array}{c}1.811 \\
(0.092)\end{array}$ & $\begin{array}{c}1.811 \\
(0.092)\end{array}$ & $2.337(0.166)$ & $\begin{array}{c}1.822 \\
(0.144)\end{array}$ & $\begin{array}{c}1.822 \\
(0.144)\end{array}$ & $\begin{array}{c}2.747 \\
(0.157)\end{array}$ & $\begin{array}{c}2.232 \\
(0.133)\end{array}$ & $\begin{array}{c}2.232 \\
(0.133)\end{array}$ \\
\hline
\end{tabular}

The oxygen amount in wine was shown in figure 5. The amount of dissolved oxygen in wines $(0.85-9.49 \mathrm{mg} / \mathrm{L})$ was similar to amounts reported in the literature (Dimkou et al., 2011; Ghidossi et al., 2012). During storage, the oxygen amount was decreasing in all studied wines and packaging. This is in accordance with earlier studies (Vidal and Moutounet, 2006; Dimkou et al., 2011). This also agrees with the $\mathrm{SO}_{2}$ decrease shown in figure 3 and 4 . Furthermore, oxygen decrease was mostly pronounced after 3 months of the storage, and after that period the changes in oxygen amounts were slower. This is in accordance with some other studies where it was stated that the decrease in oxygen amount was faster during the first months of storage (Dimkou $\boldsymbol{e t}$ al., 2011; Vidal and Moutounet, 2011; Toussaint et al., 2014). This suggests that at the beginning of the storage period, most of the oxygen was consumed and the
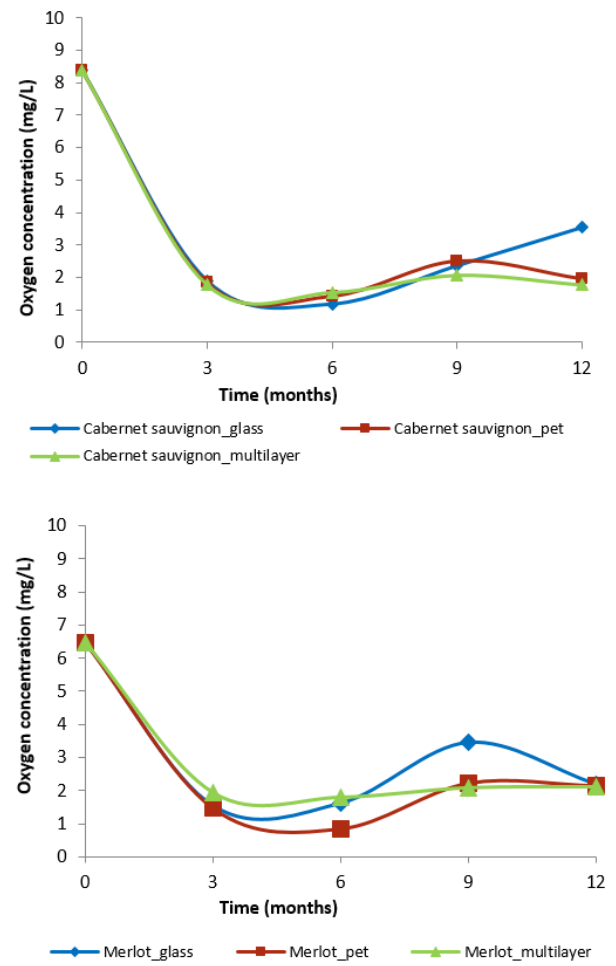

Figure 5 Oxygen concentration during storage of 12 months at $25^{\circ} \mathrm{C}$

Table 3 showed the color of wine, its intensity, hue, percentage of yellow, red and blue color. The color intensity and hue were increasing during storage in all packaging, the same as the yellow color percentage. But the percentage of red and blue color were decreasing. Statistical analysis with multiple regression (Table S1) confirms that those characteristics change with time. Namely, the increase in color intensity was statistically significant (only in Merlot packed in multilayer container, a decrease was shown). The increase of hue and yellow percentage with time in all packaging was also statistically significant (the biggest increase in these color parameters was shown for Merlot wine in multilayer containers). The decrease in red and blue percentage were also statistically significant. Fitted values of color parameters together with standard errors was showed in table 4.

Earlier studies also showed that the yellow color increases with storage (Lopes $\boldsymbol{e}$ al., 2009; Hopfer et al., 2012). The increase in hue and decrease in red color shown in our study was also shown in an earlier study (Lopes et al., 2009) Measuring A at $420 \mathrm{~nm}$ (yellow color) is a helpful tool for the determination of wine development and the degree of oxidation (Lopes et al., 2009). Namely, the increase in yellow color has been attributed to the formation of brown pigments from the polymerization of flavanols and dimeric cyanidins (Francia-Aricha $\boldsymbol{e}$ al. 1998; Drinkine et al. 2007). Similar reactions might have occurred in wines in this study.

In general, oxygen is an important factor for the wine quality. It can be dissolved in the wine before bottling or it can come from the headspace of the container. changes in wine were occurring. After that period, the oxygen consumption becomes lower. Further changes can depend mostly on the packaging material. If the oxygen permeates through the package, the oxygen amount will slowly increase and further changes in the wine will occur. In our study, further increase of oxygen amount was somewhat higher for wines packed in glass (Cabernet sauvignon and Pinot noir, which might be due to closure, and not the glass material), or it was similar for all packaging materials. After multiple regression (Table S1) the statistically significant difference in oxygen amount during the storage period of one year was shown. The analysis also confirmed the differences in the oxygen amount in wines packed in glass containers. Fitted values of oxygen amounts together with standard errors was shown in table 2.
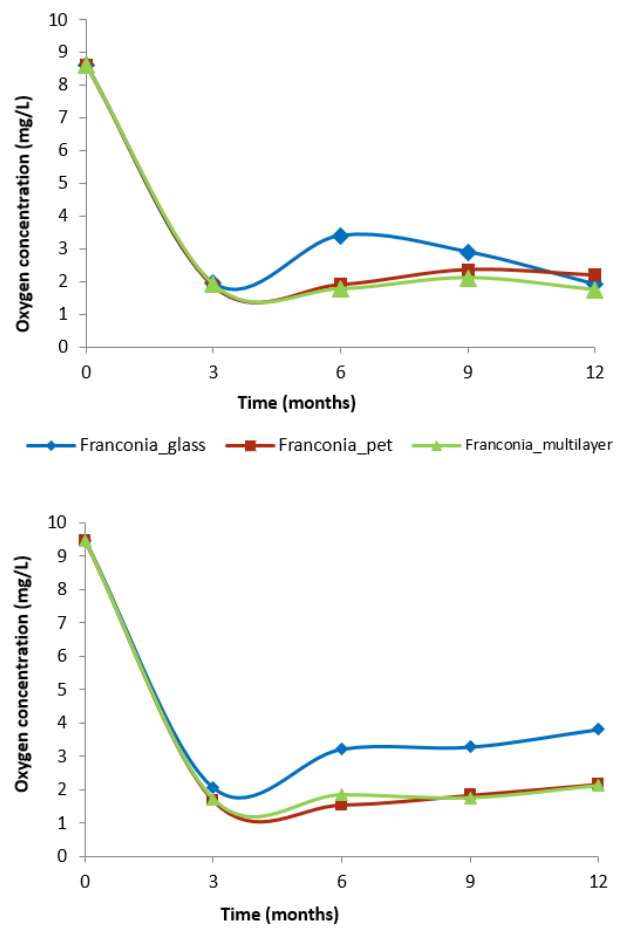

During storage, the oxygen amount decreases, because oxygen is consumed through oxidation reactions by various compounds (Dimkou et al., 2011; Ghidossi et al., 2012). Oxidation processes can be seen through changes in wine, such as changes in the wine color or $\mathrm{SO}_{2}$ decrease. Namely, the products of oxidation reactions can cause $\mathrm{SO}_{2}$ to decrease (Dimkou $\boldsymbol{e t}$ al., 2011; Ghidossi $\boldsymbol{e}$ al., 2012). The oxygen and free and total $\mathrm{SO}_{2}$ decrease was shown in our study in all wines and packaging types, which suggests the oxidation processes occurred in wine during the storage period. Changes in wine color were also observed in our study (color intensity, hue and yellow color increase, red and blue color decrease) which also suggests that the oxidation reaction occurred in the wine during the storage.

There were some differences in physico-chemical parameters of wine packed in different packaging materials. Namely, statistically significant changes in specific weight of wine and distillate, percentage of alcohol and total dry extract were shown in wines packed in multilayer and PET containers but not in glass packaging. Furthermore, the $\mathrm{SO}_{2}$ decrease, the changes of total and volatile acids were less pronounced in wines packed in glass and PET containers, in comparison to multilayer containers. All of this makes glass and PET containers a little bit better than multilayer packaging materials. It can be added that in all wines, the $\mathrm{SO}_{2}$ was above $10 \mathrm{mg} / \mathrm{L}$ at the end of storage period, which suggests that all three types of packaging could maintain the wine quality in a period of one year. 
Table 3 Color of wine during storage of 12 months at $25^{\circ} \mathrm{C}$

\begin{tabular}{|c|c|c|c|c|c|c|c|c|c|c|c|c|}
\hline \multirow{2}{*}{$\begin{array}{l}\text { Time } \\
\text { (months) }\end{array}$} & \multicolumn{3}{|c|}{ Cabernet sauvignon } & \multicolumn{3}{|c|}{ Frankovka } & \multicolumn{3}{|c|}{ Merlot } & \multicolumn{2}{|c|}{ Pinot noir } & \multirow[b]{2}{*}{$\begin{array}{l}\text { Multi } \\
\text { layer }\end{array}$} \\
\hline & Glass & PET & $\begin{array}{l}\text { Multi } \\
\text { layer }\end{array}$ & Glass & PET & $\begin{array}{l}\text { Multi } \\
\text { layer }\end{array}$ & Glass & PET & $\begin{array}{l}\text { Multi } \\
\text { layer }\end{array}$ & Glass & PET & \\
\hline \multicolumn{13}{|c|}{ Color intensity (CI) } \\
\hline $\mathbf{0}$ & 7.24 & 7.24 & 7.24 & 5.64 & 5.64 & 5.64 & 8.42 & 8.42 & 8.42 & 4.64 & 4.64 & 4.64 \\
\hline 3 & 8.56 & 8.92 & 9.25 & 6.16 & 7.26 & 7.95 & 9.85 & 9.54 & 6.35 & 5.47 & 5.51 & 5.63 \\
\hline 6 & 8.39 & 8.92 & 9.49 & 7.60 & 7.74 & 7.51 & 9.74 & 9.49 & 3.58 & 7.44 & 5.76 & 5.93 \\
\hline 9 & 8.24 & 10.09 & 8.11 & 6.61 & 8.13 & 7.01 & 8.79 & 9.79 & 4.04 & 8.65 & 6.37 & 6.23 \\
\hline 12 & 7.52 & 8.39 & 8.77 & 7.53 & 7.39 & 7.38 & 9.19 & 8.59 & 3.17 & 6.46 & 6.06 & 5.51 \\
\hline \multicolumn{13}{|c|}{ Hue (H) } \\
\hline $\mathbf{0}$ & 0.845 & 0.845 & 0.845 & 0.926 & 0.926 & 0.926 & 0.826 & 0.826 & 0.826 & 1.386 & 1.386 & 1.386 \\
\hline 3 & 1.036 & 1.085 & 1.096 & 0.957 & 0.972 & 0.931 & 1.068 & 1.014 & 0.861 & 1.362 & 1.313 & 1.295 \\
\hline 6 & 0.991 & 1.025 & 1.070 & 1.099 & 1.116 & 1.070 & 1.009 & 0.989 & 1.079 & 1.437 & 1.381 & 1.353 \\
\hline 9 & 0.948 & 1.207 & 0.911 & 1.123 & 1.198 & 0.999 & 1.046 & 1.033 & 1.259 & 1.131 & 1.356 & 1.260 \\
\hline 12 & 1.246 & 1.026 & 1.080 & 1.110 & 1.179 & 1.133 & 1.006 & 1.025 & 1.487 & 1.376 & 1.420 & 1.459 \\
\hline \multicolumn{13}{|c|}{ Yellow \% } \\
\hline 0 & 38.963 & 38.963 & 38.963 & 41.680 & 41.680 & 41.680 & 35.933 & 35.933 & 35.933 & 49.256 & 49.256 & 49.256 \\
\hline 3 & 43.554 & 44.180 & 44.334 & 42.864 & 43.089 & 40.614 & 41.303 & 38.792 & 36.704 & 49.552 & 48.829 & 48.295 \\
\hline 6 & 41.852 & 43.022 & 44.237 & 46.245 & 46.570 & 45.249 & 40.606 & 40.116 & 43.945 & 52.694 & 50.902 & 49.975 \\
\hline 9 & 40.592 & 47.260 & 39.534 & 47.101 & 48.050 & 43.220 & 41.565 & 42.794 & 45.583 & 40.335 & 50.267 & 47.936 \\
\hline 12 & 49.774 & 43.536 & 44.823 & 46.269 & 47.962 & 46.429 & 41.460 & 43.238 & 49.321 & 51.797 & 51.734 & 51.843 \\
\hline \multicolumn{13}{|c|}{ Red \% } \\
\hline 0 & 46.084 & 46.084 & 46.084 & 45.016 & 45.016 & 45.016 & 43.482 & 43.482 & 43.482 & 35.528 & 35.528 & 35.528 \\
\hline 3 & 42.036 & 40.704 & 40.441 & 44.812 & 44.328 & 43.631 & 38.665 & 38.268 & 42.628 & 36.387 & 37.194 & 37.287 \\
\hline 6 & 42.234 & 41.957 & 41.351 & 42.089 & 41.726 & 42.281 & 40.257 & 40.569 & 40.737 & 36.679 & 36.849 & 36.950 \\
\hline 9 & 42.838 & 39.166 & 43.417 & 41.938 & 40.116 & 43.249 & 39.722 & 41.446 & 36.204 & 35.676 & 37.060 & 38.040 \\
\hline 12 & 39.960 & 42.452 & 41.505 & 41.676 & 40.677 & 40.981 & 41.232 & 42.167 & 33.175 & 37.639 & 36.443 & 35.537 \\
\hline \multicolumn{13}{|c|}{ Blue \% } \\
\hline 0 & 14.953 & 14.953 & 14.953 & 13.304 & 13.304 & 13.304 & 20.585 & 20.585 & 20.585 & 15.216 & 15.216 & 15.216 \\
\hline 3 & 14.409 & 15.115 & 15.225 & 12.323 & 12.583 & 15.755 & 20.032 & 22.94 & 20.668 & 14.061 & 13.977 & 14.418 \\
\hline 6 & 15.914 & 15.021 & 14.412 & 11.666 & 11.704 & 12.470 & 19.137 & 19.315 & 15.318 & 10.627 & 12.248 & 13.076 \\
\hline 9 & 16.570 & 13.574 & 17.049 & 10.961 & 11.834 & 13.531 & 18.712 & 15.759 & 18.213 & 23.988 & 12.673 & 14.024 \\
\hline 12 & 10.266 & 14.012 & 13.672 & 12.055 & 11.361 & 12.590 & 17.309 & 14.595 & 17.504 & 10.564 & 11.823 & 12.620 \\
\hline
\end{tabular}

Table 4 Fited values of wine color and their standard errors (SE) obtained by using multiple regression with selected interactions

\begin{tabular}{|c|c|c|c|c|c|c|c|c|c|c|c|c|}
\hline \multirow{2}{*}{$\begin{array}{l}\text { Time } \\
\text { (months) }\end{array}$} & \multicolumn{2}{|c|}{ Cabernet sauvignon } & \multicolumn{3}{|c|}{ Frankovka } & \multicolumn{3}{|c|}{ Merlot } & \multicolumn{3}{|c|}{ Pinot noir } & \multirow[b]{2}{*}{ Multilayer } \\
\hline & Glass & PET & Multilayer & Glass & PET & Multilayer & Glass & PET & Multilayer & Glass & PET & \\
\hline \multicolumn{13}{|c|}{ Color intensity (CI) } \\
\hline \multirow[t]{2}{*}{$\mathbf{0}$} & 7.612 & 7.612 & 7.612 & 6.109 & 6.109 & 6.109 & 7.650 & 7.650 & 5.871 & 4.568 & 4.568 & 6.347 \\
\hline & $(0.395)$ & $(0.395)$ & $(0.395)$ & $(0.322)$ & $(0.322)$ & $(0.322)$ & $(0.403)$ & $(0.403)$ & $(0.470)$ & $(0.403)$ & $(0.403)$ & $(0.470)$ \\
\hline \multirow[t]{2}{*}{3} & 9.035 & 9.035 & 8.546 & 7.533 & 7.533 & 7.044 & 9.074 & 9.074 & 6.806 & 5.992 & 5.992 & 7.282 \\
\hline & $(0.304)$ & $(0.304)$ & $(0.288)$ & $(0.201)$ & $(0.201)$ & $(0.177)$ & $(0.314)$ & $(0.314)$ & $(0.385)$ & $(0.314)$ & $(0.314)$ & $(0.385)$ \\
\hline \multirow[t]{2}{*}{6} & 9.035 & 9.035 & 8.058 & 7.533 & 7.533 & 6.555 & 9.074 & 9.074 & 6.317 & 5.992 & 5.992 & 6.793 \\
\hline & $(0.304)$ & $(0.304)$ & $(0.318)$ & $(0.201)$ & $(0.201)$ & $(0.222)$ & $(0.314)$ & $(0.314)$ & $(0.408)$ & $(0.314)$ & $(0.314)$ & $(0.408)$ \\
\hline \multirow[t]{2}{*}{9} & 9.035 & 9.035 & 7.569 & 7.533 & 7.533 & 6.067 & 9.074 & 9.074 & 5.829 & 5.992 & 5.992 & 6.305 \\
\hline & $(0.304)$ & $(0.304)$ & $(0.382)$ & $(0.201)$ & $(0.201)$ & $(0.307)$ & $(0.314)$ & $(0.314)$ & $(0.460)$ & $(0.314)$ & $(0.314)$ & $(0.460)$ \\
\hline \multirow[t]{2}{*}{12} & 9.035 & 9.035 & $7.08(0.467)$ & 7.533 & 7.533 & 5.578 & 9.074 & 9.074 & 5.340 & 5.992 & 5.992 & 5.816 \\
\hline & $(0.304)$ & $(0.304)$ & & $(0.201)$ & $(0.201)$ & $(0.408)$ & $(0.314)$ & $(0.314)$ & $(0.532)$ & $(0.314)$ & $(0.314)$ & $(0.532)$ \\
\hline \multirow{3}{*}{ 0 } & & & & & & Hue (H) & & & & & & \\
\hline & 0.9110 & 0.9110 & 0.9110 & 0.9383 & 0.9383 & 0.9383 & 0.8863 & 0.8863 & 0.8863 & 1.2474 & 1.2474 & 1.2474 \\
\hline & $(0.0339)$ & $(0.0339)$ & (0.0339) & $(0.0339)$ & $(0.0339)$ & $(0.0339)$ & $(0.0342)$ & $(0.0342)$ & $(0.0342)$ & $(0.0339)$ & $(0.0339)$ & $(0.0339)$ \\
\hline \multirow[t]{2}{*}{3} & 1.0436 & 1.0436 & 1.0436 & 1.0708 & 1.0708 & 1.0708 & 1.0189 & 1.0189 & 1.0189 & 1.3799 & 1.3799 & 1.3799 \\
\hline & $(0.0247)$ & $(0.0247)$ & $(0.0247)$ & $(0.0247)$ & $(0.0247)$ & $(0.0247)$ & $(0.0256)$ & $(0.0256)$ & $(0.0256)$ & $(0.0247)$ & $(0.0247)$ & $(0.0247)$ \\
\hline \multirow[t]{2}{*}{6} & 1.0436 & 1.0436 & 1.0436 & 1.0708 & 1.0708 & 1.0708 & 1.0189 & 1.0189 & 1.0189 & 1.3799 & 1.3799 & 1.3799 \\
\hline & $(0.0247)$ & $(0.0247)$ & $(0.0247)$ & $(0.0247)$ & $(0.0247)$ & $(0.0247)$ & $(0.0256)$ & $(0.0256)$ & $(0.0256)$ & $(0.0247)$ & $(0.0247)$ & $(0.0247)$ \\
\hline \multirow[t]{2}{*}{9} & 1.0436 & 1.0436 & 1.0436 & 1.0708 & 1.0708 & 1.0708 & 1.0189 & 1.0189 & 1.0189 & 1.3799 & 1.3799 & 1.3799 \\
\hline & $(0.0247)$ & $(0.0247)$ & $(0.0247)$ & $(0.0247)$ & $(0.0247)$ & $(0.0247)$ & $(0.0256)$ & $(0.0256)$ & $(0.0256)$ & $(0.0247)$ & $(0.0247)$ & $(0.0247)$ \\
\hline \multirow[t]{2}{*}{12} & 1.0436 & 1.0436 & 1.0436 & 1.0708 & 1.0708 & 1.0708 & 1.0189 & 1.0189 & 1.4870 & 1.3799 & 1.3799 & 1.3799 \\
\hline & $(0.0247)$ & $(0.0247)$ & $(0.0247)$ & $(0.0247)$ & $(0.0247)$ & $(0.0247)$ & $(0.0256)$ & $(0.0256)$ & $(0.0927)$ & $(0.0247)$ & $(0.0247)$ & $(0.0247)$ \\
\hline \multirow{3}{*}{ 0 } & & & & & & Yellow \% & & & & & & \\
\hline & 41.90 & 41.90 & $41.90(0.47)$ & 41.90 & 41.90 & $41.90(0.47)$ & 35.89 & $35.89(0.70)$ & $35.89(0.70)$ & 47.91 & 47.91 & $47.91(0.70)$ \\
\hline & $(0.47)$ & $(0.47)$ & & $(0.47)$ & $(0.47)$ & & $(0.70)$ & & & $(0.70)$ & $(0.70)$ & \\
\hline \multirow[t]{2}{*}{3} & 42.92 & 42.92 & $42.92(0.34)$ & 42.92 & 42.92 & $42.92(0.34)$ & 38.28 & $38.28(0.52)$ & $38.28(0.52)$ & 48.94 & 48.94 & $48.94(0.58)$ \\
\hline & $(0.34)$ & $(0.34)$ & & $(0.34)$ & $(0.34)$ & & $(0.52)$ & & & $(0.58)$ & $(0.58)$ & \\
\hline 6 & 43.95 & 43.95 & $43.95(0.32)$ & 43.95 & 43.95 & $43.95(0.32)$ & 40.67 & $40.67(0.50)$ & $40.67(0.50)$ & 49.96 & 49.96 & $49.96(0.51)$ \\
\hline & $(0.32)$ & $(0.32)$ & & $(0.32)$ & $(0.32)$ & & $(0.50)$ & & & $(0.51)$ & $(0.51)$ & \\
\hline 9 & 44.98 & 44.98 & $44.98(0.42)$ & 44.98 & 44.98 & $44.98(0.42)$ & 43.07 & $43.07(0.67)$ & $43.07(0.67)$ & 40.34 & 50.99 & $50.99(0.53)$ \\
\hline & $(0.42)$ & $(0.42)$ & & $(0.42)$ & $(0.42)$ & & $(0.67)$ & & & $(2.10)$ & $(0.53)$ & \\
\hline 12 & 46.00 & 46.00 & $46.00(0.59)$ & 46.00 & 46.00 & $46.00(0.59)$ & 45.46 & $45.46(0.92)$ & $45.46(0.92)$ & 52.02 & 52.02 & $52.02(0.62)$ \\
\hline & $(0.59)$ & $(0.59)$ & & $(0.59)$ & $(0.59)$ & & $(0.92)$ & & & $(0.62)$ & $(0.62)$ & \\
\hline & & & & & & Red \% & & & & & & \\
\hline $\mathbf{0}$ & 44.291 & 44.291 & 44.291 & 44.707 & 44.707 & 44.707 & 44.533 & 44.533 & 44.533 & 36.579 & 36.579 & 36.579 \\
\hline & $(0.507)$ & $(0.507)$ & $(0.507)$ & $(0.507)$ & $(0.507)$ & $(0.507)$ & $(0.716)$ & $(0.716)$ & $(0.716)$ & $(0.716)$ & $(0.716)$ & $(0.716)$ \\
\hline 3 & 41.953 & 41.953 & 41.953 & 42.369 & 42.369 & 42.369 & 39.886 & 39.886 & 39.886 & 36.549 & 36.549 & 36.549 \\
\hline & $(0.369)$ & $(0.369)$ & $(0.369)$ & $(0.369)$ & $(0.369)$ & $(0.369)$ & $(0.407)$ & $(0.407)$ & $(0.407)$ & $(0.390)$ & $0.390)$ & $(0.390)$ \\
\hline 6 & 41.953 & 41.953 & 41.953 & 42.369 & 42.369 & 42.369 & 39.886 & 39.886 & 39.886 & 36.549 & 36.549 & 36.549 \\
\hline & $(0.369)$ & $(0.369)$ & $(0.369)$ & $(0.369)$ & $(0.369)$ & $(0.369)$ & $(0.407)$ & $(0.407)$ & $(0.407)$ & $(0.390)$ & $(0.390)$ & $(0.390)$ \\
\hline 9 & 41.953 & 41.953 & 41.953 & 42.369 & 42.369 & 42.369 & 39.886 & 39.886 & 39.886 & 36.549 & 36.549 & 36.549 \\
\hline & $(0.369)$ & $(0.369)$ & (0.369) & $(0.369)$ & $(0.369)$ & $(0.369)$ & $(0.407)$ & $(0.407)$ & $(0.407)$ & $(0.390)$ & $(0.390)$ & $(0.390)$ \\
\hline 12 & 41.953 & 41.953 & 41.953 & 42.369 & 42.369 & 42.369 & 39.886 & 39.886 & 33.175 & 36.549 & 36.549 & 36.549 \\
\hline & $(0.369)$ & $(0.369)$ & $(0.369)$ & $(0.369)$ & $(0.369)$ & $(0.369)$ & $(0.407)$ & $(0.407)$ & (1.386) & $(0.390)$ & $(0.390)$ & $(0.390)$ \\
\hline & & & & & & Blue \% & & & & & & \\
\hline $\mathbf{0}$ & 16.201 & 16.201 & 16.201 & 13.935 & 13.935 & 13.935 & 20.102 & 20.102 & 20.102 & 14.567 & 14.567 & 14.567 \\
\hline
\end{tabular}




\begin{tabular}{|c|c|c|c|c|c|c|c|c|c|c|c|c|}
\hline & $(0.307)$ & $(0.307)$ & $(0.307)$ & $(0.423)$ & $(0.423)$ & $(0.423)$ & $(0.423)$ & $(0.423)$ & $(0.423)$ & $(0.426)$ & $(0.426)$ & $(0.426)$ \\
\hline \multirow[t]{2}{*}{3} & 15.503 & 15.503 & 15.503 & 13.237 & 13.237 & 13.237 & 19.404 & 19.404 & 19.404 & 13.869 & 13.869 & 13.869 \\
\hline & $(0.217)$ & $(0.217)$ & $(0.217)$ & $(0.362)$ & $(0.362)$ & $(0.362)$ & $(0.362)$ & $(0.362)$ & $(0.362)$ & $(0.369)$ & $(0.369)$ & $(0.369)$ \\
\hline \multirow[t]{2}{*}{6} & 14.806 & 14.806 & 14.806 & 12.539 & 12.539 & 12.539 & 18.706 & 18.706 & 18.706 & 13.171 & 13.171 & 13.171 \\
\hline & $(0.179)$ & $(0.179)$ & $(0.179$ & $(0.339)$ & (0.339) & $(0.339)$ & $(0.339)$ & $(0.339)$ & $(0.339)$ & $(0.350)$ & $(0.350)$ & $(0.350)$ \\
\hline \multirow[t]{2}{*}{9} & 14.108 & 14.108 & 14.108 & 11.841 & 11.841 & 11.841 & 18.009 & 18.009 & 18.009 & 23.988 & 12.474 & 12.474 \\
\hline & $(0.220)$ & $(0.220)$ & $(0.220)$ & $(0.362)$ & $(0.362)$ & $(0.362)$ & $(0.362)$ & $(0.362)$ & $(0.362)$ & $(1.372)$ & $(0.375)$ & $(0.375)$ \\
\hline \multirow[t]{2}{*}{12} & 13.410 & 13.410 & 13.410 & 11.143 & 11.143 & 11.143 & 17.311 & 17.311 & 17.311 & 11.776 & 11.776 & 11.776 \\
\hline & $(0.310)$ & $(0.310)$ & $(0.310)$ & $(0.422)$ & $(0.422)$ & $(0.422)$ & $(0.422)$ & $(0.422)$ & $(0.422)$ & $(0.436)$ & $(0.436)$ & $(0.436)$ \\
\hline
\end{tabular}

\section{CONCLUSION}

The results showed that the physicochemical properties of wine are influenced by the storage time and packaging material. Namely, some small changes with time in specific weight of wine and distillate, alcohol percentage and dry extract were shown. On the other hand, the concentration of sulfur dioxide and oxygen decreased significantly over storage time. The color also showed statistically significant change with storage time (the color intensity, hue and yellow percentage showed an increased with storage period, blue and red color, on the other hand, decreased). This suggests the oxidation processes occurred in wine during the storage period. Changes in wine parameters with packaging type were also shown. The specific weight of wine and distillate, alcohol percentage, and total dry extract showed small changes in wines packed in PET and multilayer containers but not in glass bottles. The $\mathrm{SO}_{2}$ amount decrease was lower in wines packed in glass and PET containers. According to these results, it can be suggested that glass and PET packaging are somewhat better for wine than multilayer packaging.

Acknowledgements: Authors are very grateful to Kolar family cellar and winery Pinkert for giving wine samples for examination. We would also like to thank to prof. Andrew R. Barron (Yale University, USA) for his help in statistical analysis of the data.

\section{REFERENCES}

Caillé, S., Samson, A., Wirth, J., Diéval, J., Vidal, S., \& Cheynier, V. (2010) Sensory characteristics changes of red Grenache wines submitted to different oxygen exposures pre and post bottling. Analytica chimica acta, 660(1-2), 35-42 http://doi.org/10.1016/j.aca.2009.11.049

Dimkou, E., Ugliano, M., Diéval, J. B., Vidal, S., Aagaard, O., Rauhut, D., \& Jung, R. (2011). Impact of headspace oxygen and closure on sulfur dioxide, color, and hydrogen sulfide levels in a Riesling wine. American Journal of $\begin{array}{llll}\text { Enology and } & \text { V2(3), } & \text { 261-269. }\end{array}$ http://dx.doi.org/10.5344/ajev.2011.11006

Drinkine, J., Lopes, P., Kennedy, J. A., Teissedre, P.-L., \& Saucier, C. (2007) Ethylidene-bridged flavan-3-ols in red wine and correlation with wine age Journal of Agricultural and Food Chemistry, 55(15), 6292-6299. https://doi.org/10.1021/jf070038w

Francia-Aricha, E. M., Rivas-Gonzalo, J. C., \& Santos-Buelga, C. (1998). Effect of malvidin-3-monoglucoside on the browning of monomeric and dimeric flavanols. Zeitschrift für Lebensmitteluntersuchung Und-Forschung A, 207(3), 223-228. https://doi.org/10.1007/s002170050323

Gambuti, A., Rinaldi, A., Ugliano, M., \& Moio, L. (2013). Evolution of Phenolic Compounds and Astringency during Aging of Red Wine:Effect of Oxygen Exposure before and after Bottling. Journal of Agricultural and Food Chemistry, 61(8), 1618-1627. https://doi.org/10.1021/jf302822b

Ghidossi, R., Poupot, C., Thibon, C., Pons, A., Darriet, P., Riquier, L., De Revel G., \& Mietton-Peuchot, M. (2012). The influence of packaging on wine $\begin{array}{llll}\text { conservation. } & \text { Food } & \text { Control, } & \text { 23(2), }\end{array}$ https://doi.org/10.1016/j.foodcont.2011.06.003

Glories, Y. (1984). La couleur des vins rouges. 2e partie: mesure, origine et interprétation. OENO One, 18(4), 253-271. https://doi.org/10.20870/oenoone.1984.18.4.1744

Hopfer, H., Ebeler, S. E., \& Heymann, H. (2012). The combined effects of storage temperature and packaging type on the sensory and chemical properties of Chardonnay. Journal of Agricultural and Food Chemistry, 60(43), 10743 10754. https://doi.org/10.1021/jf302910f

International Organisation of Vine and Wine. (2007). Compendium of International Methods of Wine and Must Analysis, Volume 1. OIV, Paris, France: http://www.oiv.int/public/medias/6619/compendium-2019-en-vol1.pdf.

Kojić, N., \& Jakobek, L. (2019). Chemical and sensory properties of red wines from Baranja vineyards. In: S Tomas, Đ Ačkar (eds.). Proceedings of International Conference $17^{\text {th }}$ Ružička days "Today Science - Tomorrow industry”, 63-71.

Lopés, P., Silva, M. A., Pons, A., Tominaga, T., Lavigne, V., Saucier, C., Darriet, P., Teissedre, P. - L., \& Dubourdieu, D. (2009). Impact of oxygen dissolved at bottling and transmitted through closures on the composition and sensory properties of a Sauvignon blanc wine during bottle storage. Journal of Agricultural and Food Chemistry, 57(21), 10261-10270. https://doi.org/10.1021/jf9023257

Monagas, M., Gómez-Cordovés, C. \& Bartolomé, B. (2005). Evolution of polyphenols in red wines from Vitis vinifera L. during aging in the bottle
European Food Research and Technology, 220, 607-614. https://doi.org/10.1007/s00217-004-1108-x

Robertson, G. L. (1993). Aseptic Packaging of Foods. In: Food packaging: principles and practice, 381-408. Marcel Dekker, New York.

Skouroumounis, G. K., Kwiatkowski, M. J., Francis, I. L., Oakey, H., Capone, D. L., Duncan, B., Sefton, M. A., \& Waters, E. J. (2005). The impact of closure type and storage conditions on the composition, colour and flavour properties of a Riesling and a wooded Chardonnay wine during five years' storage. Australian Journal of Grape and Wine Research, 11(3), 369-377. https://doi.org/10.1111/j.1755-0238.2005.tb00036.x

Toussaint, M., Vidal, J. - C., \& Salmon, J. - M. (2014). Comparative Evolution of Oxygen, Carbon Dioxide, Nitrogen, and Sulfites during Storage of a Rosé Wine Bottled in PET and Glass. Journal of Agricultural and Food Chemistry, 62(13), 2946-2955. https://doi.org/10.1021/jf405392u

Vidal, J. - C., \& Moutounet, M. (2006). Monitoring of oxygen in the gas and liquide phases of bottles of wine at bottling and during storage. OENO One, 40(1), 35-45. https://doi.org/10.20870/oeno-one.2006.40.1.884

Vidal, J. - C., \& Moutounet, M. (2011). Impact of operating conditions during bottling and of technical cork permeabiliy on the oxygen content and evolution of bottled Sauvignon blanc. Internet Journal of Viticulture and Enology, 4/3, 1-15. 
Table S1 The results of the multiple regression with the selected strongly significant $(\mathrm{p}<0.001)$ main effects and interactions for all physico-chemical parameters and color in wine

\begin{tabular}{|c|c|c|c|}
\hline Term & Coefficient & $\begin{array}{c}\begin{array}{c}\text { Standard error of } \\
\text { coefficient }\end{array} \\
\end{array}$ & P-Value \\
\hline \multicolumn{4}{|l|}{$\begin{array}{l}\text { PHYSICO-CHEMICAL PROPERTIES OF } \\
\text { WINE }\end{array}$} \\
\hline & \multicolumn{3}{|c|}{ Specific weight of wine } \\
\hline Constant & 0.994228 & 0.000035 & 0,000 \\
\hline Merlot $v s$ Pinot noir & 0.000925 & 0.000092 & 0,000 \\
\hline multilayer $v s$ time & 0.000049 & 0.000008 & 0.000 \\
\hline PET vs time & -0.000029 & 0.000007 & 0.000 \\
\hline Merlot $v s$ time & -0.000042 & 0.000011 & 0.000 \\
\hline Merlot : multilayer : time at least 6 months & -0.001384 & 0.000148 & 0.000 \\
\hline & \multicolumn{3}{|c|}{ Specific weight of distillate } \\
\hline Constant & 0.981557 & 0.000024 & 0.000 \\
\hline multilayer vs PET & 0.000526 & 0.000060 & 0.000 \\
\hline Cabernet $v s$ others & -0.001307 & 0.000056 & 0.000 \\
\hline Frankovka vs Merlot and Pinot noir & 0.001554 & 0.000059 & 0.000 \\
\hline multilayer $v s$ PET : time 0 months $v s$ others & -0.000658 & 0.000148 & 0.000 \\
\hline multilayer vs PET :-time 3 months vs larger & -0.000810 & 0.000153 & 0.000 \\
\hline Merlot $v s$ Pinot noir : time & -0.000087 & 0.000010 & 0.000 \\
\hline Merlot : multilayer : time 12 months & 0.000778 & 0.000204 & 0.000 \\
\hline & \multicolumn{3}{|c|}{ Alcohol (\% vol.) } \\
\hline Constant & 14.2434 & 0.0217 & 0.000 \\
\hline multilayer vs PET & -0.4587 & 0.0534 & 0.000 \\
\hline Cabernet $v s$ others & 1.1759 & 0.0498 & 0.000 \\
\hline Frankovka $v s$ Merlot and Pinot noir & -1.3782 & 0.0530 & 0.000 \\
\hline multilayer $v s$ PET : time 0 months $v s$ others & 0.573 & 0.132 & 0.000 \\
\hline multilayer vs PET : time 3 months vs larger & 0.745 & 0.137 & 0.000 \\
\hline Merlot $v s$ Pinot noir : time & 0.07942 & 0.00870 & 0.000 \\
\hline Merlot : multilayer : time 12 months & -0.746 & 0.182 & 0.000 \\
\hline & \multicolumn{3}{|c|}{ Total dry extract (g/L) } \\
\hline Constant & 32.6671 & 0.0681 & 0.000 \\
\hline Merlot $v s$ Pinot noir & 3.335 & 0.198 & 0.000 \\
\hline Cabernet $v s$ others & 3.679 & 0.154 & 0.000 \\
\hline Frankovka vs Merlot and Pinot noir & -3.481 & 0.165 & 0.000 \\
\hline Merlot : multilayer : time at least 6 months & -4.508 & 0.331 & 0.000 \\
\hline & \multicolumn{3}{|c|}{ Total acid (g/L) } \\
\hline Constant & 5.7937 & 0.0212 & 0.000 \\
\hline Frankovka $v s$ Merlot and Pinot noir & 0.9277 & 0.0519 & 0.000 \\
\hline Merlot $v s$ Pinot noir & 0.582 & 0.0599 & 0.000 \\
\hline & \multicolumn{3}{|c|}{ Volatile acid (g/L) } \\
\hline Constant & 0.53906 & 0.00799 & 0.000 \\
\hline Cabernet $v s$ others & 0.1765 & 0.01550 & 0.000 \\
\hline Frankovka $v s$ Merlot and Pinot noir & -0.0884 & 0.02340 & 0.000 \\
\hline Frankovka $v s$ time & 0.01101 & 0.00297 & 0.000 \\
\hline Multilayer : time & 0.0063 & 0.00168 & 0.000 \\
\hline & \multicolumn{3}{|c|}{ Free $\mathrm{SO}_{2}(\mathrm{mg} / \mathrm{L})$} \\
\hline Constant & 16.872 & 0.372 & 0.000 \\
\hline Merlot vs Pinot noir & -5.71 & 1.04 & 0.000 \\
\hline time 0 months vs others & 20.379 & 0.925 & 0.000 \\
\hline $\begin{array}{r}\text { Frankovka } v s \text { Merlot and Pinot noir : time } 0 \\
\text { months } v s \text { larger }\end{array}$ & 14.01 & 2.27 & 0.000 \\
\hline \multirow{2}{*}{$\begin{array}{r}\text { months } v s \text { larger } \\
\text { Frankovka : glass : time } 3 \text { months }\end{array}$} & 14.67 & 2.91 & 0.000 \\
\hline & \multicolumn{3}{|c|}{ Total $\mathrm{SO}_{2}(\mathrm{mg} / \mathrm{L})$} \\
\hline Constant & 38.394 & 0.563 & 0.000 \\
\hline Cabernet $v s$ others & -7.2 & 1.27 & 0.000 \\
\hline Merlot vs Minot noir & 9.82 & 1.62 & 0.000 \\
\hline time 0 months vs others & 56.63 & 1.36 & 0.000 \\
\hline time 3 months $v s$ larger & 22.95 & $\mathbf{1 . 5 3}$ & 0.000 \\
\hline Cabernet $v s$ others : time 3 months $v s$ larger & -19.08 & 3.33 & 0.000 \\
\hline Merlot $v s$ Pinot noir-time 0 months $v s$ larger & 23.97 & 3.88 & 0.000 \\
\hline Merlot $v s$ Pinot noir-time 3 months $v s$ larger & 36.16 & 4.34 & 0.000 \\
\hline Merlot : glass : time 6 months & 41.07 & 4.39 & 0.000 \\
\hline Merlot : multilayer : time 3 months & -43.13 & 4.97 & 0.000 \\
\hline Frankovka : multilayer : time 3 months & -18.96 & 4.48 & 0.000 \\
\hline \multirow[t]{2}{*}{ Pinot noir : PET : time-9 months } & 17.06 & 4.39 & 0.000 \\
\hline & \multicolumn{3}{|c|}{ Oxygen $(\mathrm{mg} / \mathrm{L})$} \\
\hline Constant & 3.3203 & 0.0679 & 0.000 \\
\hline time 0 months vs others & 6.690 & 0.196 & 0.000 \\
\hline glass $v s$ others & 0.516 & 0.144 & 0.001 \\
\hline Merlot $v s$ Pinot noir & -0.843 & 0.192 & 0.000 \\
\hline Merlot : time 0 months $v s$ others & -2.162 & 0.392 & 0.000 \\
\hline
\end{tabular}




\section{COLOR OF WINE}

Constant $\quad 7.624$

Cabernet $v s$ others $\quad 1.502$

Merlot vs Pinot noir $\quad 3.082$

time 0 months $v s$ others $\quad \mathbf{- 1 . 4 2 4}$

Merlot $v s$ Pinot noir : multilayer $\quad-3.558$

Multilayer : time $\quad \mathbf{- 0 . 1 6 2 9}$

Constant $\quad 1.1018$

time 0 months $v s$ others

Merlot vs Pinot noir

Cabernet $v s$ others

Frankovka $v s$ Merlot and Pinot noir

Merlot : multilayer : time 12 months

\section{Constant}

Merlot vs Pinot noir

Cabernet $v s$ others

Frankovka vs Merlot and Pinot noir

time 0 months $v s$ others

Merlot vs Pinot noir : time 0 months vs larger Merlot : multilayer : time 12 months

$\mathbf{- 0 . 1 3 2 6}$

$-0.3610$

$-0.1130$

$-0.1286$

0.4681

40.657

4.260

2.352

4.152

2.338

4.62

$-6.71$

Constant

Merlot vs Pinot noir

time

Merlot:time

Pinot noir : glass : time 9 months

41.899

$-12.03$

0.3420

0.456

$-10.66$

Constant

Merlot vs Pinot noir

time

16.201

5.535

$\mathbf{- 0 . 2 3 2 6}$

Frankovka vs Merlot and Pinot noir

Pinot noir : glass : time 9 months

$-3.400$

$\mathbf{1 1 . 5 1}$

Color intensity (CI)

$0.160 \quad 0.000$

$0.322 \quad 0.000$

$0.484 \quad 0.000$

$\begin{array}{ll}0.362 & 0.000\end{array}$

$0.838 \quad 0.000$

$\begin{array}{ll}0.0387 & 0.000\end{array}$

Hue (H)

$0.0121 \quad 0.000$

$\begin{array}{ll}0.0300 & 0.000\end{array}$

$0.0345 \quad 0.000$

$0.0277-0.000$

$0.0295 \quad 0.000$

$0.0962 \quad 0.000$

$\begin{array}{ll}\text { Yellow (\%) } & \\ 0.181 & 0.000\end{array}$

$\begin{array}{ll}0.515 & 0.000\end{array}$

$0.415 \quad 0.000$

$0.441 \quad 0.000$

$0.448 \quad 0.000$

$1.27 \quad 0.001$

$\begin{array}{ll}1.44 & 0.000\end{array}$

$\operatorname{Red}(\%)$

$0.470-0.000$

$1.05 \quad 0.000$

$\begin{array}{ll}0.0703 & 0.000\end{array}$

$0.115 \quad 0.000$

$\begin{array}{ll}2.17 & 0.000\end{array}$

Blue (\%)

$\begin{array}{ll}0.307 & 0.000\end{array}$

$0.510 \quad 0.000$

$\begin{array}{ll}0.0419 & 0.000\end{array}$

$0.436 \quad 0.000$

1.42

$\mathbf{0 . 0 0 0}$

Regression main effect terms are built from indicators of the indicated condition ( 1 if true and 0 otherwise). Main effect orthogonal contrasts $(v s)$ are built from differences of multiples of indicators (such that the coefficient would be the difference in the means, when the other terms are orthogonal thereto). Interaction terms (marked with :) are built from products of these. Time as a continuous variable is also considered, with care in subset selection as it is not orthogonal to the other contrasts. Term selection is by preservation of strong significance ( $p<0.001)$, set especially small to avoid large models and because we are not otherwise accounting for multiple comparisons. Three way interaction terms appear only when residual analysis of the best two way interaction model reveals large standardized residuals (magnitude $>3$ ) at the corresponding measurement, such that no such large residuals occur in the final model. Terms are shown in bold when they correspond to time changes as a main effect or time changes in interaction with the type of packaging. 